\title{
Densely arrayed cage-shaped polymer topologies synthesized via cyclopolymerization of star-shaped macromonomers
}

Yoshinobu Mato, ${ }^{\dagger}$ Maho Sudo, ${ }^{\dagger}$ Hironori Marubayashi, ${ }^{\S}$ Brian J. Ree, ${ }^{\ddagger}$ Kenji Tajima, ${ }^{\ddagger}$ Takuya Yamamoto, ${ }^{\ddagger}$ Hiroshi Jinnai, ${ }^{\S}$ Takuya Isono, ${ }^{\ddagger},{ }^{*}$ Toshifumi Satoh ${ }^{\ddagger *}$

${ }^{\dagger}$ Graduate School of Chemical Sciences and Engineering, Hokkaido University, Sapporo 060-8628, Japan

${ }^{7}$ Division of Applied Chemistry, Faculty of Engineering, Hokkaido University, Sapporo 060-8628, Japan

§Institute of Multidisciplinary Research for Advanced Materials, Tohoku University, 2-1-1 Katahira, Aoba-ku, Sendai, Miyagi 980-8577, Japan 


\section{Table of Contents}

S1. Experimental Section 3

S1-1. Materials 3

S1-2. Instruments $\quad 5$

S1-3. Synthetic details 10

S2. Additional Results $\quad 25$

S3. References $\quad 40$ 


\section{S1. Experimental Section}

\section{S1-1. Materials}

Macromonomers (NB-PCL-NB, $s-(\mathrm{PCL}-\mathrm{NB})_{3}$, and $\left.s-(\mathrm{PCL}-\mathrm{NB})_{4}\right)$ and monomeric PCL samples (PCL-NB, c-PCL, cage $_{3}$-PCL, and cage $_{4}-\mathrm{PCL}$ ) were prepared according to our reported papers. ${ }^{1,2}$ Grubbs' catalyst $3^{\text {rd }}$ generation (G3) was prepared according to the previously reported method. ${ }^{3}$ Amberlyst $^{\circledR}$ A21 (Organo Co., Ltd.), N,N-dimethyl-4-aminopyridine (DMAP; Tokyo Chemical Industry Co., Ltd. (TCI), >99.0\%), 1-ethyl-3-(3-(dimethylamino)-propyl)carbodiimide hydrochloride (EDC; TCI, >98.0\%), ethyl vinyl ether (TCI, >98.0\%), ( \pm )-exo-5-norbornenecarboxylic acid (exo-NB-COOH; Aldrich, 97\%), sodium methoxide (NaOMe; $1 \mathrm{~mol} \mathrm{~L}^{-1}$ in methanol, Kanto Chemical Co., Inc.), Dowex ${ }^{\circledR}$ 50WX2 hydrogen form (Aldrich, 50-100 mesh), sodium iodide (NaI; Wako Pure Chemical Industry Co. Ltd., >99.5\%), 2,5-dihydroxybenzoic acid (DHB; Sigma-Aldrich, $>98.0 \%$ ), dithranol (Fluka, $>98 \%$ ), sodium trifluoroacetate (ACROS Organics, $97 \%$ ), $\mathrm{CH}_{2} \mathrm{Cl}_{2}(\mathrm{Junsei}$ Chemical Co., Ltd., >99.0\%), diethyl ether (Kanto Chemical Co., Inc., >99\%), methanol (MeOH; Kanto Chemical Co., Inc., $>99.5 \%$ ), and dry- $\mathrm{CH}_{2} \mathrm{Cl}_{2}$ (Kanto Chemical Co., Inc., $>99.5 \%$, water content, $<0.001 \%$ ) were used as received. Dry-toluene (Kanto Chemical Co., Inc., $>99.5 \%$, water content, $<0.001 \%$ ) was further purified by an MBRAUN MB SPS Compact solvent purification system equipped with a MB-KOL-C and a MB-KOL-A columns, which were directly used for reactions. $\varepsilon^{-}$ Caprolactone ( $\varepsilon$-CL; TCI, >99\%), 1-butanol (Wako Pure Chemical Industry Co. Ltd., >99.0\%), and tin(II) ethylhexanoate (Aldrich, 95\%) were purified by distillation over $\mathrm{CaH}_{2}$ under reduced pressure 
and stored in the glovebox. L-Lactide (TCI, $>98.0 \%$ ) was purified twice by recrystallization using dry toluene and stored in the glovebox. Trimethylene carbonate was kindly received from Mitsubishi Chemical Co., Ltd and purified by recrystallization using dry tetrahydrofuran and diethyl ether, followed by kept in the glovebox. 1,3,5-trihydroxymethylbenzene (TCI, >95\%), pentaerythritol (TCI, $>98.0 \%$ ), and diphenyl phosphate (DPP; TCI, $>99.0 \%$ ) were purified by azeotropic distillation with dry-toluene and stored in the glovebox. Four-armed star-shaped poly(ethylene glycol) with a hydroxyl group at each chain end (4-Arm PEG-OH 5k Da, namely $s$-(PEG-OH) 4 ; MW $=5,160 \mathrm{~g} \mathrm{~mol}^{-1}$, dispersity $=1.03,>99.9 \%$ ) was purchased from Creative PEGWorks and purified by reprecipitation from $\mathrm{CH}_{2} \mathrm{Cl}_{2}$ to cold diethyl ether before use. 


\section{S1-2. Instruments}

The polymerization experiments were carried out in an MBRAUN stainless steel glovebox equipped with a gas purification system (molecular sieves and copper catalyst) in a dry argon atmosphere $\left(\mathrm{H}_{2} \mathrm{O}, \mathrm{O}_{2}<0.1 \mathrm{ppm}\right)$. The moisture and oxygen contents in the glovebox were monitored by an MB-MO-SE 1 moisture sensor and an MB-OX-SE 1 oxygen sensor, respectively. The ${ }^{1} \mathrm{H}$ NMR (400 MHz) spectra were recorded using a JEOL JNM-ECS400 instrument at room temperature in $\mathrm{CDCl}_{3}$. The size exclusion chromatography (SEC) was performed at $40{ }^{\circ} \mathrm{C}$ in THF (flow rate, $1.0 \mathrm{~mL}$ $\min ^{-1}$ ) using a Shodex GPC-101 gel permeation chromatography system (Shodex DU-2130 dual pump, Shodex RI-71-S reflective index detector, and Shodex ERC-3125SN degasser) equipped with a Shodex KF-G guard column $(4.6 \mathrm{~mm} \times 10 \mathrm{~mm}$; particle size, $8 \mu \mathrm{m})$ and two Shodex KF-804L columns (linear, $8 \mathrm{~mm} \times 300 \mathrm{~mm}$ ) or Jasco high-performance liquid chromatography system (PU-3 980 Intelligent HPLC Pump, CO-2065 Plus Intelligent Column Oven, RI-2031 Plus Intelligent RI Detector, and DG2080-53 Degasser) equipped with a Shodex KF-G guard column (4.6 mm $\times 10 \mathrm{~mm}$; particle size, 8 $\mu \mathrm{m})$ and two Shodex KF-804L columns (linear; particle size $7 \mu \mathrm{m} ; 8.0 \mathrm{~mm} \times 300 \mathrm{~mm}$; exclusion limit, $4 \times 10^{4}$ ) or in DMF (flow rate, $0.6 \mathrm{~mL} \mathrm{~min}^{-1}$; containing $0.01 \mathrm{~mol} \mathrm{~L}^{-1} \mathrm{LiCl}$ ) using a JASCO HPLC system (PU-980 Intelligent HPLC Pump, CO-965 Column Oven, RI-930 Intelligent RI Detector, and DG-2080-53 Degasser) equipped with a Shodex KD-G guard column (4.6 mm × $10 \mathrm{~mm}$; particle size, $8 \mu \mathrm{m}$ ), a Shodex Asahipak GF-310 HQ column (linear; particle size, $5 \mu \mathrm{m} ; 7.5 \mathrm{~mm} \times 300 \mathrm{~mm}$; exclusion limit, $4 \times 10^{4}$ ) and a Shodex Asahipak GF-7 M HQ column (linear; particle size, $9 \mu \mathrm{m}$; 7.5 
$\mathrm{mm} \times 300 \mathrm{~mm}$; exclusion limit, $\left.1.0 \times 10^{7}\right)$. The number-average molecular weight $\left(M_{\mathrm{n}, \mathrm{SEC}}\right)$ and the dispersity $(Ð)$ of the polymers were calculated on the basis of polystyrene calibrations. The absolute number-averaged and weight-averaged molecular weights ( $M_{\mathrm{n}, \mathrm{MALS}}$ and $M_{\mathrm{w}, \mathrm{MALS}}$, respectively) of the samples were determined by SEC with multiangle light scattering detection (SEC-MALS-Visco) in THF (flow rate, $1.0 \mathrm{~mL} \mathrm{~min}{ }^{-1}$ ) at $40{ }^{\circ} \mathrm{C}$ using an Agilent 1100 series instrument equipped with a DG 1100 degasser, a Shodex KF-G guard column (4.6 mm $\times 10 \mathrm{~mm}$; particle size, $8 \mu \mathrm{m})$, a Shodex KF800D solvent-peak separation column (linear, $8.0 \mathrm{~mm} \times 100 \mathrm{~mm}$; particle size, $10 \mu \mathrm{m}$ ), two Shodex KF-805L columns (linear, $8.0 \mathrm{~mm} \times 300 \mathrm{~mm}$; exclusion limit, $4.0 \times 10^{6}$; particle size, $10 \mu \mathrm{m}$ ), a DAWN 8+ multiangle laser light scattering detector (Wyatt Technology), an Optilab rEX refractive index detector (Wyatt Technology), and a Viscostar viscosity detector (Wyatt Technology). The preparative SEC for Grubbs' catalyst removal was performed at r.t. in $\mathrm{CHCl}_{3}$ (flow rate, 3.5 and $10 \mathrm{~mL} \mathrm{~min}^{-1}$ ) using LC-9201 liquid chromatography system (Japan Analytical Industry Co. Ltd.) equipped with a BG-12 degasser, a PI-50 pump, a RI-50S RI detector, a JAIGEL-H-P guard column $(8 \mathrm{~mm} \times 40 \mathrm{~mm}$; Japan Analytical Industry Co. Ltd.), and a Shodex K-2004 column (linear, $20.0 \mathrm{~mm} \times 300 \mathrm{~mm}$; exclusion limit, $1.4 \times 10^{4}$; particle size, $7 \mu \mathrm{m}$ ) or LaboACE LC-7080 liquid chromatography system (Japan Analytical Industry Co. Ltd.) equipped with a JAIGEL-HR-P guard column (8 $\mathrm{mm} \times 40 \mathrm{~mm}$; Japan Analytical Industry Co. Ltd.) and JAIGEL-4HR $(20 \mathrm{~mm} \times 600 \mathrm{~mm}$; exclusion limit, 5× 105; Japan Analytical Industry Co. Ltd.). 


\section{Matrix-assisted laser desorption ionization time-of-flight mass spectrometry (MALDI-}

TOF MS). MALDI-TOF MS of the obtained polymers was performed using an Applied Biosystems Voyager-DE STR-H equipped with a $337 \mathrm{~nm}$ nitrogen laser (3 ns pulse width). Two hundred shots were accumulated for the spectra at a $20 \mathrm{kV}$ acceleration voltage in the reflector or linear mode and calibrated using PSt as the internal standard. PCL samples for the MALDI-TOF MS were prepared as follows: (i) the THF solution of polymer sample $\left(4.0 \mathrm{mg} \mathrm{mL}^{-1}\right)$ and the THF solution of matrix (DHB; $60 \mathrm{mg} \mathrm{mL}^{-1}$ ) were mixed at a volume ratio of $1: 1$. (ii) Then, the sample plate was spotted by the THF solution of cationic agent $\left(\mathrm{NaI} ; 1.0 \mathrm{mg} \mathrm{mL}^{-1}, 1.0 \mu \mathrm{L}\right)$, followed by the mixed solution $(1.0 \mu \mathrm{L})$. The methanolyzed polynorbornene sample was prepared by depositing a mixture $(1.0 \mu \mathrm{L})$ of polymer $(10$ $\mathrm{mg} \mathrm{mL} \mathrm{m}^{-1}, 100 \mu \mathrm{L}$ in THF), matrix (dithranol; $20 \mathrm{mg} \mathrm{mL}^{-1}, 20 \mu \mathrm{L}$ in THF), and cationic agent (sodium trifluoroacetate; $20 \mathrm{mg} \mathrm{mL}^{-1}, 20 \mu \mathrm{L}$ in $\mathrm{MeOH}$ ).

Differential Scanning Calorimetry (DSC). DSC analysis was carried out on a DSC7000X (Hitachi High-Tech Corporation) calibrated with the indium and tin standards. The system was purged with nitrogen gas at a flow rate of $50 \mathrm{~mL} \mathrm{~min}^{-1}$. All analyses were performed by utilizing 3-7 $\mathrm{mg}$ of samples in hermetically sealed aluminum pans. The isothermal crystallization studies to determine the equilibrium melting temperature $\left(T_{\mathrm{m}}{ }^{\circ}\right)$ were carried out according to the Hoffman-Weeks extrapolation method. ${ }^{4}$ The samples were heated on a hot plate (EYELA VOM-1000) from room temperature to $100{ }^{\circ} \mathrm{C}$ and kept at $100{ }^{\circ} \mathrm{C}$ for 5 min under vacuum to eliminate thermal history. Then, the samples were cooled down rapidly to a constant crystallization temperature $\left(T_{\mathrm{c}} ; 0-40{ }^{\circ} \mathrm{C}\right)$ using a 
cooling plate (AS ONE SCP-85). At that temperature, the samples were held for adequate time (more than $40 \mathrm{~h}$ ). After the isothermal steps, the samples were immediately immersed in liquid $\mathrm{N}_{2}$ to quit the crystallization and heated again up to $100{ }^{\circ} \mathrm{C}$ at the heating rate of $10^{\circ} \mathrm{C} \mathrm{min}^{-1}$ to record the melting temperature $\left(T_{\mathrm{m}}\right)$. The determined $T_{\mathrm{m}}$ values for the isothermally crystallized samples were plotted against $T_{\mathrm{c}} \mathrm{S}$, and their linear extrapolation to the line of $T_{\mathrm{m}}=T_{\mathrm{c}}$ gave $T_{\mathrm{m}}{ }^{\circ}$ as the intersection.

\section{Small-angle X-ray scattering (SAXS) and wide-angle X-ray diffraction (WAXD) analysis.}

Synchrotron SAXS and WAXD measurements of the obtained polymers were performed with an Xray beam of a wavelength $(\lambda)$ of $1.500 \AA$ at the BL-6A in the Photon Factory of KEK (Tsukuba, Japan). The 2D SAXS and WAXD patterns were obtained with PILATUS3 1M and PILATUS 100K detectors, respectively, which were circularly averaged to produce the $1 \mathrm{D}$ plots of $I$ (scattering intensity with an arbitrary unit) and $q$ [scattering vector; $q=4 \pi \sin \theta / \lambda$, where $\theta$ is a half of scattering angle $(2 \theta)]$. The $q$ value was calibrated using silver behenate. The samples were heated on a hot plate (EYELA VOM-1000) from room temperature to $100^{\circ} \mathrm{C}$ and kept at $100^{\circ} \mathrm{C}$ for 5 min under vacuum to eliminate thermal history. Then, the samples were cooled down rapidly to $30^{\circ} \mathrm{C}$ and held for $48 \mathrm{~h}$ using a cooling plate (AS ONE SCP-85). The obtained powder sample was sandwiched by two pieces of Kapton tapes with a spacer of a stainless washer, which was applied for the measurement. The crystallinity ( $\left.X_{\mathrm{WAXD}}\right)$ was determined by the peak separation of WAXD profile. The lamellae thickness $\left(l_{\mathrm{c}}\right)$, amorphous layer thickness $\left(l_{\mathrm{a}}\right)$, and long period $\left(L_{\mathrm{p}}\right)$ were evaluated by the correlation function analysis of SAXS profile 
as follows: Each SAXS curve was converted to the normalized one-dimensional correlation function $[\gamma(r)]:^{5}$

$$
\gamma(r)=\frac{\int_{0}^{\infty} q^{2} I(q) \cos (q r) \mathrm{d} q}{\int_{0}^{\infty} q^{2} I(q) \mathrm{d} q}
$$

where $r$ is the correlation length (nm). Integral calculations were executed for $0 \leq q \leq q$ cutoff, where $q_{\text {cutoff }}$ is the scattering vector at which the calculated value of $q^{2} I(q)$ became a sufficiently small value. $I(q)$ in a relatively low $q$ range $\left(0 \leq q \leq 0.2-0.4 \mathrm{~nm}^{-1}\right)$ was calculated according to the Guinier law ${ }^{6}$ and $I(q)$ in a relatively high $q$ range $\left(q>1.1-1.6 \mathrm{~nm}^{-1}\right)$ was determined on the basis of the Porod law. Based on the lamellar two-phase model, $l_{\mathrm{c}}, l_{\mathrm{a}}$, and $L_{\mathrm{p}}$ were determined from $\gamma(r)$ by the method of Strobl and Schneider. ${ }^{7}$ 


\section{S1-3. Synthetic details}

\section{Synthesis of PCL-OH}

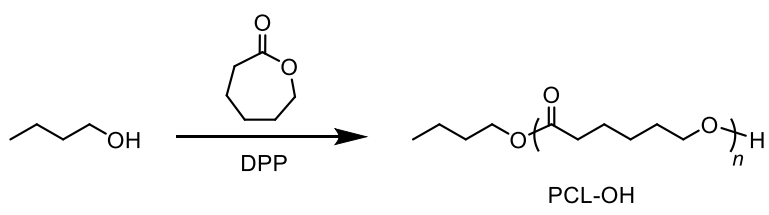

In the glovebox, $\varepsilon$-CL (4.00 g, $35.0 \mathrm{mmol})$, 1-butanol (173 mg, $2.34 \mathrm{mmol})$, and DPP (53.4 $\mathrm{mg}, 213 \mu \mathrm{mol})$ were added to a reaction vessel. The reaction mixture was stirred at $80{ }^{\circ} \mathrm{C}$ for $18 \mathrm{~min}$. The polymerization was quenched by the addition of Amberlyst ${ }^{\circledR} A 21$. The polymer crude was purified by the reprecipitation from $\mathrm{CH}_{2} \mathrm{Cl}_{2}$ into cold $\mathrm{MeOH}$ to give PCL-OH as a white solid $(2.48 \mathrm{~g})$. Yield: $70.2 \%$

$M_{\mathrm{n}, \mathrm{NMR}}=1,790 \mathrm{~g} \mathrm{~mol}^{-1}, M_{\mathrm{n}, \mathrm{SEC}}=2,810 \mathrm{~g} \mathrm{~mol}^{-1}, Ð=1.11$

${ }^{1} \mathrm{H}$ NMR (400 MHz, $\left.\mathrm{CDCl}_{3}\right): \delta(\mathrm{ppm})$ 4.10-4.03 (m, -OCO $\left.\left(\mathrm{CH}_{2}\right)_{4} \mathrm{CH}_{2}-\right), 3.67$ (q, 2H, $J=6.10$, $\left.\mathrm{CH}_{2} \mathrm{OH}\right), \quad 2.37-2.26 \quad\left(\mathrm{~m}, \quad-\mathrm{OCOCH}_{2}\left(\mathrm{CH}_{2}\right)_{4}-\right), \quad 1.75-1.54 \quad\left(\mathrm{~m}, \quad-\mathrm{OCOCH}_{2} \mathrm{CH}_{2}\left(\mathrm{CH}_{2}\right)_{3}{ }^{-}, \quad-\right.$ $\left.\mathrm{OCO}\left(\mathrm{CH}_{2}\right)_{3} \mathrm{CH}_{2} \mathrm{CH}_{2}-\right), 1.50-1.30\left(\mathrm{~m},-\mathrm{OCO}\left(\mathrm{CH}_{2}\right)_{2} \mathrm{CH}_{2}\left(\mathrm{CH}_{2}\right)_{2}-,-\mathrm{CH}_{2} \mathrm{CH}_{2} \mathrm{CH}_{2} \mathrm{CH}_{3},-\mathrm{CH}_{2} \mathrm{CH}_{2} \mathrm{CH}_{2} \mathrm{CH}_{3}\right.$, $\left.-\mathrm{CH}_{2} \mathrm{CH}_{2} \mathrm{CH}_{2} \mathrm{CH}_{3}\right), 0.94\left(\mathrm{t}, 3 \mathrm{H}, J=7.30,-\mathrm{CH}_{3}\right)$. 


\section{Synthesis of PCL-NB}

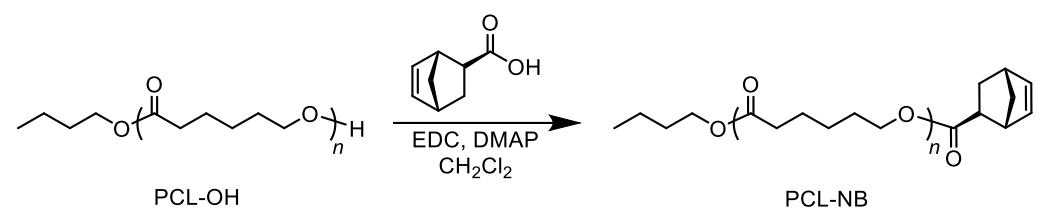

A typical procedure for the condensation reaction is as follows (Method A): In a Schlenk flask,

PCL-OH $\left(M_{\mathrm{n}, \mathrm{NMR}}=1,790 \mathrm{~g} \mathrm{~mol}^{-1}, 2.20 \mathrm{~g}, 1.22 \mathrm{mmol}\right)$, exo-NB-COOH (340 mg, $\left.2.46 \mathrm{mmol}\right)$, EDC (708 mg, $3.69 \mathrm{mmol})$, and DMAP (450 mg, $3.68 \mathrm{mmol})$ were dissolved in dry $\mathrm{CH}_{2} \mathrm{Cl}_{2}(22.0 \mathrm{~mL})$ and the mixture was stirred at r.t. for $24 \mathrm{~h}$. The polymer crude was purified by reprecipitation twice from $\mathrm{CH}_{2} \mathrm{Cl}_{2}$ into cold $\mathrm{MeOH}$ to give PCL-NB as a white solid (1.57 g). Yield: $66.2 \%$

$M_{\mathrm{n}, \mathrm{NMR}}=2,040 \mathrm{~g} \mathrm{~mol}^{-1}, M_{\mathrm{n}, \mathrm{SEC}}=2,970 \mathrm{~g} \mathrm{~mol}^{-1}, Ð=1.12$

${ }^{1} \mathrm{H}$ NMR (400 MHz, $\left.\mathrm{CDCl}_{3}\right): \delta(\mathrm{ppm})$ 6.23-6.07 (m, -CH=CH- in norbornene ring), 4.17-3.99 (m, $\mathrm{OCO}\left(\mathrm{CH}_{2}\right)_{4} \mathrm{CH}_{2}$ ), 3.03 (s, - $\mathrm{CH}-\mathrm{CH}-\mathrm{COO}-$ in norbornene ring), 2.92 (s, - $\mathrm{CH}-\mathrm{CH}_{2}-\mathrm{CH}-\mathrm{COO}-$ in norbornene ring), 2.39-2.25 (m, $\left.-\mathrm{OCOCH}_{2}\left(\mathrm{CH}_{2}\right)_{4}-\right)$, 2.25-2.19 (m, endo- $\mathrm{CH}-$ of - $\left.\mathrm{CH}-\mathrm{CH}_{2}-\mathrm{CH}-\mathrm{CH}_{2} \mathrm{O}-\right)$, 1.95-1.88 (m, - $\mathrm{CH}_{2}-\mathrm{CH}-\mathrm{COO}-$ in norbornene ring), $1.77-1.54$ (m, $-\mathrm{OCOCH}_{2} \mathrm{CH}_{2}\left(\mathrm{CH}_{2}\right)_{3^{-}}$, $\left.\mathrm{OCO}\left(\mathrm{CH}_{2}\right)_{3} \mathrm{CH}_{2} \mathrm{CH}_{2}-\right), 1.47-1.32\left(\mathrm{~m},-\mathrm{OCO}\left(\mathrm{CH}_{2}\right)_{2} \mathrm{CH}_{2}\left(\mathrm{CH}_{2}\right)_{2}-,-\mathrm{CH}_{2} \mathrm{CH}_{2} \mathrm{CH}_{2} \mathrm{CH}_{3},-\mathrm{CH}_{2} \mathrm{CH}_{2} \mathrm{CH}_{2} \mathrm{CH}_{3}\right.$, $\left.-\mathrm{CH}_{2} \mathrm{CH}_{2} \mathrm{CH}_{2} \mathrm{CH}_{3}\right), 0.94\left(\mathrm{t}, 3 \mathrm{H}, J=7.3,-\mathrm{CH}_{3}\right)$. 


\section{Synthesis of $l$-GPCL}

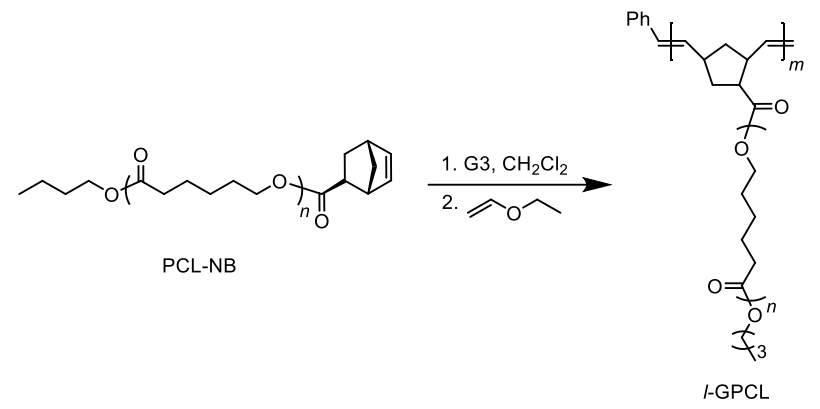

In a vial, a stock solution of $\mathrm{G} 3\left(163 \mu \mathrm{L}\right.$ as a $20 \mathrm{mmol} \mathrm{L}^{-1}$ solution in dry- $\left.\mathrm{CH}_{2} \mathrm{Cl}_{2}, 3.26 \mu \mathrm{mol}\right)$

was added to a stirred solution of PCL-NB $\left(M_{\mathrm{n}, \mathrm{NMR}}=2,040 \mathrm{~g} \mathrm{~mol}^{-1}, 100 \mathrm{mg}, 49.0 \mu \mathrm{mol}\right)$ in dry- $\mathrm{CH}_{2} \mathrm{Cl}_{2}$ $(4.9 \mathrm{~mL})$ under an argon atmosphere. After $10 \mathrm{~min}$, ethyl vinyl ether was added to reacting mixture to terminate the ROMP. The mixture was purified by reprecipitation from $\mathrm{CH}_{2} \mathrm{Cl}_{2}$ into cold $\mathrm{MeOH}$ to give $\boldsymbol{l}$-GPCL as a pale brown solid (73.6 mg). Yield: $73.5 \%$

$M_{\mathrm{n}, \mathrm{SEC}}=29,600 \mathrm{~g} \mathrm{~mol}^{-1}, Ð=1.06$

${ }^{1} \mathrm{H}$ NMR (400 MHz, $\mathrm{CDCl}_{3}$ ): $\delta$ (ppm) 5.65-4.87 (br, alkenyl of poly(norbornene) backbone), 4.39-3.74 (m, $\left.-\mathrm{OCO}\left(\mathrm{CH}_{2}\right)_{4} \mathrm{CH}_{2}-\right)$, 3.28-1.07 (br, cyclopentane ring of poly(norbornene) backbone), 2.45-2.14 (m, $\left.-\mathrm{OCOCH}_{2}\left(\mathrm{CH}_{2}\right)_{4-}\right)$, 1.83-1.49 (m, $\left.-\mathrm{OCOCH}_{2} \mathrm{CH}_{2}\left(\mathrm{CH}_{2}\right)_{3}{ }^{-},-\mathrm{OCO}\left(\mathrm{CH}_{2}\right)_{3} \mathrm{CH}_{2} \mathrm{CH}_{2-}\right), 1.46-1.24$ (m, $\left.\mathrm{OCO}\left(\mathrm{CH}_{2}\right)_{2} \mathrm{CH}_{2}\left(\mathrm{CH}_{2}\right)_{2}-,-\mathrm{CH}_{2} \mathrm{CH}_{2} \mathrm{CH}_{2} \mathrm{CH}_{3},-\mathrm{CH}_{2} \mathrm{CH}_{2} \mathrm{CH}_{2} \mathrm{CH}_{3},-\mathrm{CH}_{2} \mathrm{CH}_{2} \mathrm{CH}_{2} \mathrm{CH}_{3}\right), 0.94$ (t, $3 \mathrm{H}, J=$ $\left.7.3,-\mathrm{CH}_{3}\right)$. 


\section{Synthesis of $c$-GPCL}
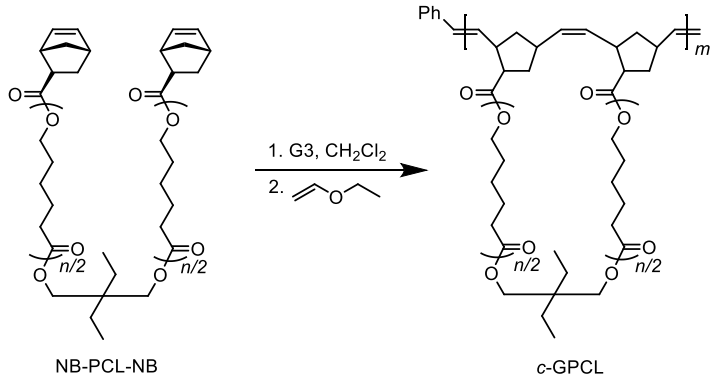

A typical procedure for the cyclopolymerization is as follows (method B): In a three-necked

flask, a stock solution of G3 $\left(521 \mu \mathrm{L}\right.$ as a $4.0 \mathrm{mM}$ solution in dry- $\left.\mathrm{CH}_{2} \mathrm{Cl}_{2}, 2.08 \mu \mathrm{mol}\right)$ was quickly added to a stirred mixture of NB-PCL-NB $\left(M_{\mathrm{n}, \mathrm{NMR}}=3,200 \mathrm{~g} \mathrm{~mol}^{-1}, 50.0 \mathrm{mg}, 15.6 \mu \mathrm{mol}\right)$ in dry$\mathrm{CH}_{2} \mathrm{Cl}_{2}(78.1 \mathrm{~mL})$ under an argon atmosphere at room temperature. After $60 \mathrm{~min}$, excess amount of ethyl vinyl ether was added to the mixture to terminate the reaction. The metal residue in the crude product was removed by preparative SEC (solvent, $\mathrm{CHCl}_{3}$ ) to give $\boldsymbol{c}$-GPCL as a pale brown solid (49.7 mg). Yield: 99.1\%

$M_{\mathrm{n}, \mathrm{SEC}}=23,700 \mathrm{~g} \mathrm{~mol}^{-1}, Ð=1.14$

${ }^{1} \mathrm{H}$ NMR (400 MHz, $\mathrm{CDCl}_{3}$ ): $\delta$ (ppm) 6.70-4.85 (br, alkenyl of poly(norbornene) backbone), 4.16-3.77 (m, - $\left.\mathrm{OCO}\left(\mathrm{CH}_{2}\right)_{4} \mathrm{CH}_{2}-\right), 3.88\left(\mathrm{~s},\left(\mathrm{CH}_{3} \mathrm{CH}_{2}\right)_{2} \mathrm{C}-\right)$, 3.42-1.73 (br, cyclopentane ring of poly(norbornene) backbone $), \quad 2.40-2.19 \quad\left(\mathrm{~m}, \quad-\mathrm{OCOCH}_{2}\left(\mathrm{CH}_{2}\right)_{4}{ }^{-}\right), \quad 1.76-1.51 \quad\left(\mathrm{~m}, \quad-\mathrm{OCOCH}_{2} \mathrm{CH}_{2}\left(\mathrm{CH}_{2}\right)_{3^{-}}\right.$, $\left.\mathrm{OCO}\left(\mathrm{CH}_{2}\right)_{3} \mathrm{CH}_{2} \mathrm{CH}_{2-}\right), 1.48-1.20\left(\mathrm{~m}, \mathrm{CH}_{3} \mathrm{CH}_{2-},-\mathrm{OCO}\left(\mathrm{CH}_{2}\right)_{2} \mathrm{CH}_{2}\left(\mathrm{CH}_{2}\right)_{2-}\right), 0.97\left(\mathrm{~s},-\mathrm{CH}_{3}\right)$. 


\section{Synthesis of cage $_{3}-$ GPCL}
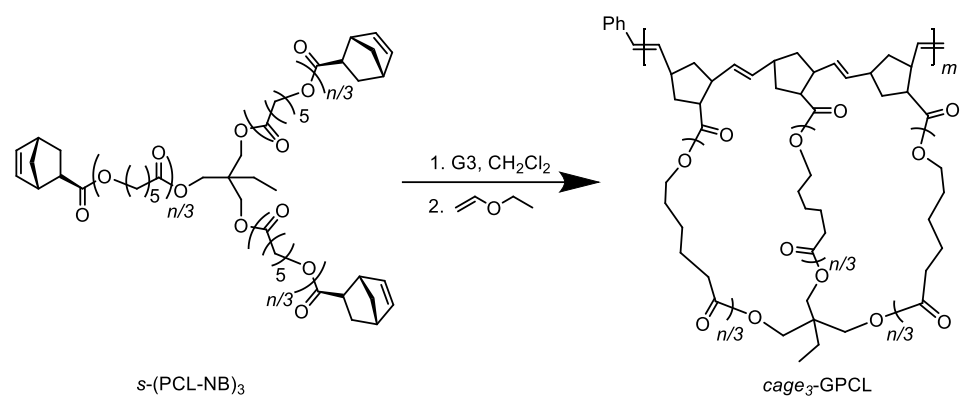

Method B was used for the cyclopolymerization of $s-(\text { PCL-NB })_{3}\left(M_{\mathrm{n}, \mathrm{NMR}}=4,700 \mathrm{~g} \mathrm{~mol}^{-1}\right.$, $50.0 \mathrm{mg}, 10.6 \mu \mathrm{mol})$ with $\mathrm{G} 3\left(532 \mu \mathrm{L}\right.$ as a $4.0 \mathrm{mM}$ solution in dry- $\left.\mathrm{CH}_{2} \mathrm{Cl}_{2}, 2.13 \mu \mathrm{mol}\right)$ in dry- $\mathrm{CH}_{2} \mathrm{Cl}_{2}$ (106 $\mathrm{mL})$ for $2 \mathrm{~h}$ to give cage $_{3}$-GPCL as a pale brown solid $(49.7 \mathrm{mg})$. Yield: $98.9 \%$

$M_{\mathrm{n}, \mathrm{SEC}}=18,200 \mathrm{~g} \mathrm{~mol}^{-1}, Ð=1.17$

${ }^{1} \mathrm{H}$ NMR (400 MHz, $\mathrm{CDCl}_{3}$ ): $\delta$ (ppm) 6.54-4.79 (br, alkenyl of poly(norbornene) backbone), 4.39-3.73 (m, - $\left.\mathrm{OCO}\left(\mathrm{CH}_{2}\right)_{4} \mathrm{CH}_{2}-\right)$, 3.23-0.95 (br, cyclopentane ring of poly(norbornene) backbone), 2.39-2.18 (m, $\left.-\mathrm{OCOCH}_{2}\left(\mathrm{CH}_{2}\right)_{4-}\right), \quad 1.74-1.50$ (m, - $\left.\mathrm{OCOCH}_{2} \mathrm{CH}_{2}\left(\mathrm{CH}_{2}\right)_{3}-,-\mathrm{OCO}\left(\mathrm{CH}_{2}\right)_{3} \mathrm{CH}_{2} \mathrm{CH}_{2}-\right), \quad 1.49-1.28$ (m, $\left.\mathrm{CH}_{3} \mathrm{C}\left(\mathrm{CH}_{2}-\right)_{3},-\mathrm{OCO}\left(\mathrm{CH}_{2}\right)_{2} \mathrm{CH}_{2}\left(\mathrm{CH}_{2}\right)_{2}-\right), 0.67-0.95\left(\mathrm{~m},-\mathrm{CH}_{3}\right)$. 


\section{Synthesis of cage $_{4}$-GPCL}
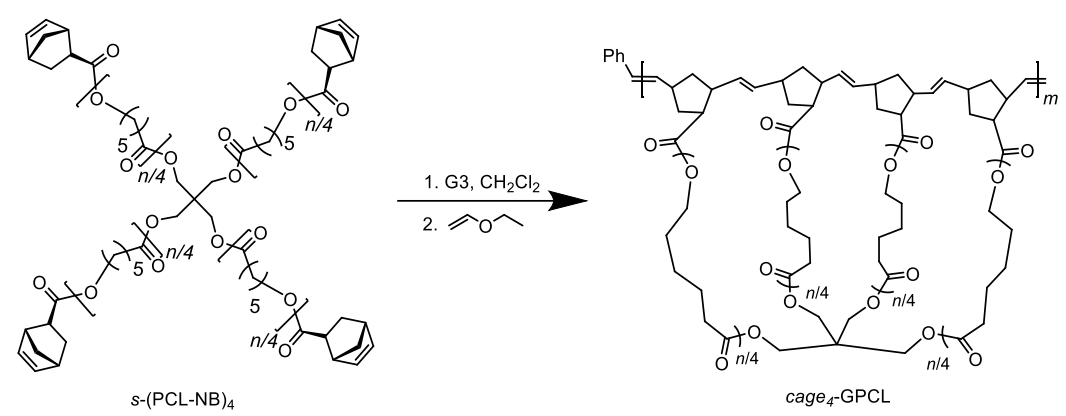

Method B was used for the cyclopolymerization of $s-(\text { PCL-NB })_{4}\left(M_{\mathrm{n}, \mathrm{NMR}}=6,000 \mathrm{~g} \mathrm{~mol}^{-1}\right.$,

$50.0 \mathrm{mg}, 8.35 \mu \mathrm{mol})$ with $\mathrm{G} 3\left(556 \mu \mathrm{L}\right.$ as a $4.0 \mathrm{mM}$ solution in dry- $\left.\mathrm{CH}_{2} \mathrm{Cl}_{2}, 2.22 \mu \mathrm{mol}\right)$ in dry- $\mathrm{CH}_{2} \mathrm{Cl}_{2}$

(41.7 $\mathrm{mL}$ ) for $90 \mathrm{~min}$ to give cage $_{4}$-GPCL as a pale brown solid $(49.5 \mathrm{mg})$. Yield: $98.8 \%$

$M_{\mathrm{n}, \mathrm{SEC}}=24,000 \mathrm{~g} \mathrm{~mol}^{-1}, Ð=1.27$

${ }^{1} \mathrm{H}$ NMR (400 MHz, $\mathrm{CDCl}_{3}$ ): $\delta$ (ppm) 6.58-4.79 (br, alkenyl of poly(norbornene) backbone), 4.42-3.75

(m, -OCO( $\left.\left.\mathrm{CH}_{2}\right)_{4} \mathrm{CH}_{2}-\right)$, 3.24-1.04 (br, cyclopentane ring of poly(norbornene) backbone), 2.42-2.21 (m, $\left.-\mathrm{OCOCH}_{2}\left(\mathrm{CH}_{2}\right)_{4-}\right)$, 1.83-1.51 (m, $\left.-\mathrm{OCOCH}_{2} \mathrm{CH}_{2}\left(\mathrm{CH}_{2}\right)_{3}-{ }^{-},-\mathrm{OCO}\left(\mathrm{CH}_{2}\right)_{3} \mathrm{CH}_{2} \mathrm{CH}_{2}-\right), \quad 1.50-1.29$ (m, $\left.\mathrm{C}\left(\mathrm{CH}_{2}-\right)_{4},-\mathrm{OCO}\left(\mathrm{CH}_{2}\right)_{2} \mathrm{CH}_{2}\left(\mathrm{CH}_{2}\right)_{2}-\right)$. 


\section{Methanolysis of cage $_{3}-$ GPCL}

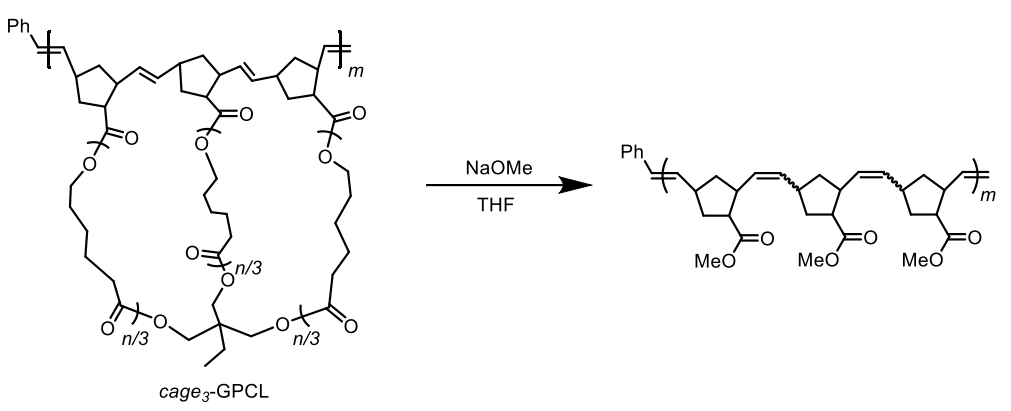

In a vial, $\mathrm{NaOMe}(870 \mu \mathrm{L}$ as $1 \mathrm{M}$ solution in $\mathrm{MeOH}, 870 \mu \mathrm{mol})$ was added to the solution of cage $_{3}$-GP $\left(M_{\mathrm{n}}=47,000\right.$, calculated by the following equation: $\left(M_{\mathrm{n}, \mathrm{NMR}}\right.$ of $\left.s-(\mathrm{PCL}-\mathrm{NB})_{3} ; 4,700\right) \times([s-$ $\left.\left.\left.(\mathrm{PCL}-\mathrm{NB})_{3}\right]_{0} /[\mathrm{G} 3]_{0} ; 10\right), 98.1 \mathrm{mg}, 2.09 \mu \mathrm{mol}\right)$ in dry-THF $(3.3 \mathrm{~mL})$, and then the solution was stirred at r.t. for 24 h. The Dowex ${ }^{\circledR} 50 \mathrm{WX} 2$ and water were added to the reaction mixture to let it neutralize. The crude product was evaporated and purified by the preparative SEC (eluent; $\mathrm{CHCl}_{3}$ ) to give poly(methyl norbornenecarboxylate) as a pale brown solid (2.9 mg). Yield: $27.4 \%$ 


\section{Synthesis of cage-GPs with diverse polymer backbones}

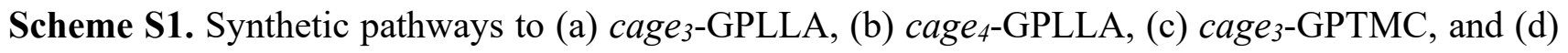
cage $_{4}$-GPEG

(a) cage $_{3}$-GPLLA

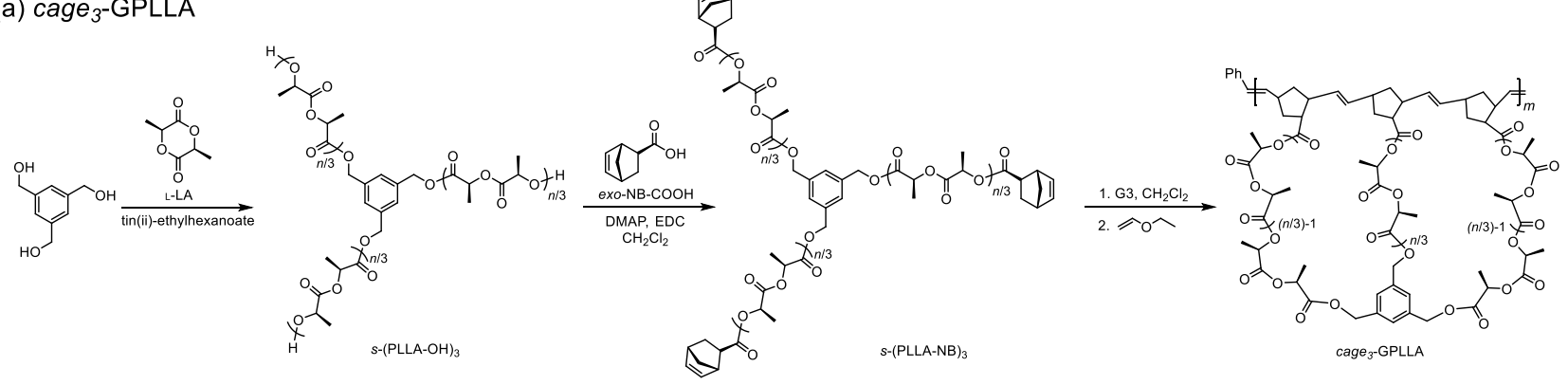

(b) cage $_{4}$-GPLLA
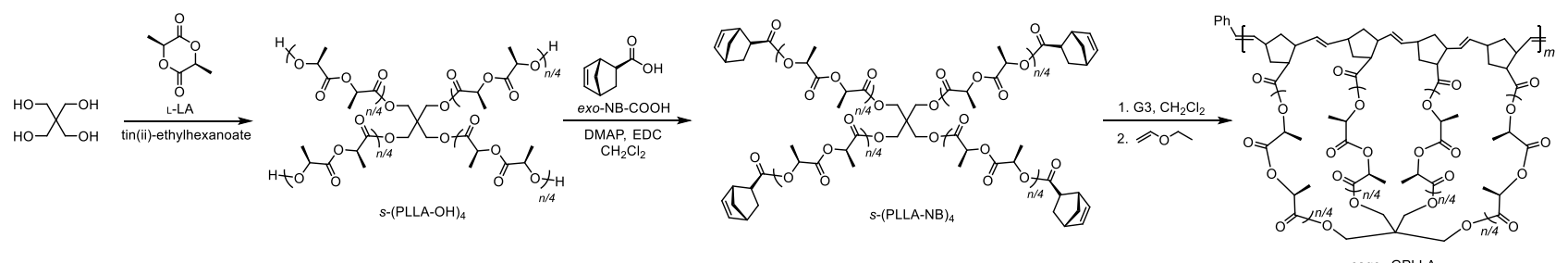

(c) cage $_{3}$-GPTMC

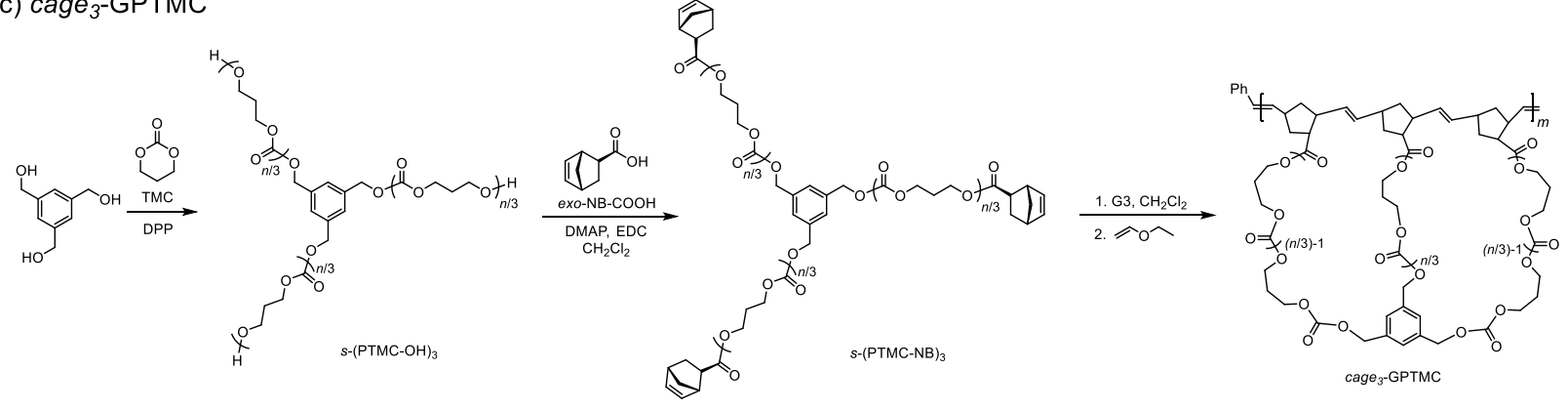

(d) cage $_{4}$-GPEG
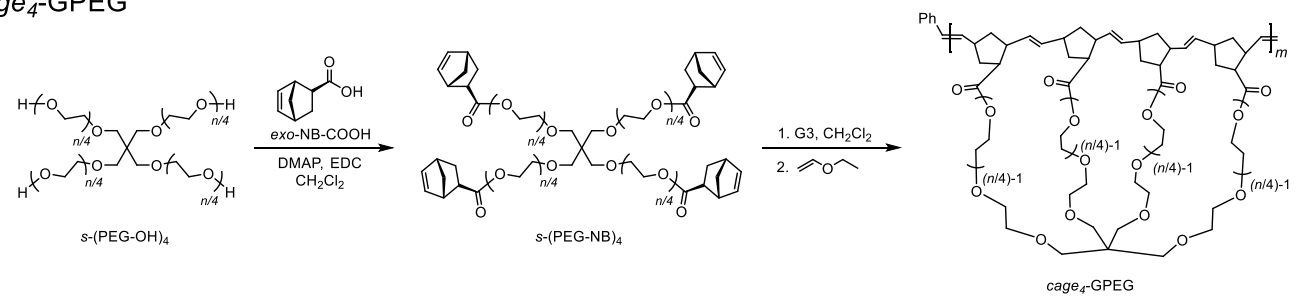


\section{Synthesis of $s-($ PLLA-OH $) 3$}

A typical procedure for the ring-opening polymerization is as follows (Method $\mathrm{C}$ ): In the glovebox, L-lactide (1.00 g, $8.76 \mathrm{mmol})$, 1,3,5-trihydroxymethylbenzene $(29.2 \mathrm{mg}, 173 \mu \mathrm{mol})$, and tin(II) ethylhexanoate $\left(7.4 \mu \mathrm{L}\right.$ as $0.1 \mathrm{~mol} \mathrm{~L}^{-1}$ solution in toluene, $\left.0.74 \mu \mathrm{mol}\right)$ were placed in a reaction vessel. The reaction mixture was stirred at $120{ }^{\circ} \mathrm{C}$ for $8.6 \mathrm{~h}$. The polymerization was quenched by the addition of excess amount of $\mathrm{CH}_{2} \mathrm{Cl}_{2}$. The polymer crude was purified by the reprecipitation from $\mathrm{CH}_{2} \mathrm{Cl}_{2}$ into cold $\mathrm{MeOH}$ to give $\boldsymbol{s}$-(PLLA-OH)3 as a white solid $(811 \mathrm{mg})$. Yield: $78.8 \%$ $M_{\mathrm{n}, \mathrm{NMR}}=6,770 \mathrm{~g} \mathrm{~mol}^{-1}, M_{\mathrm{n}, \mathrm{SEC}}=9,510 \mathrm{~g} \mathrm{~mol}^{-1}, Ð=1.13$

${ }^{1} \mathrm{H}$ NMR (400 MHz, $\left.\mathrm{CDCl}_{3}\right): \delta(\mathrm{ppm}) 7.25$ (s, aromatic), 5.29-5.06 (m, methine of PLLA backbone, $\left.\mathrm{CH}_{2} \mathrm{O}-\right), 4.44-4.27\left(\mathrm{~m},-\mathrm{CH}\left(\mathrm{CH}_{3}\right) \mathrm{OH}\right), 2.77-2.55(\mathrm{~m},-\mathrm{OH}), 1.86-1.37$ (m, methyl of PLLA backbone).

\section{Synthesis of $s-(\text { PLLA-NB })_{3}$}

Method A was used for the condensation reaction of $s$-(PLLA-OH $)_{3}\left(M_{\mathrm{n}, \mathrm{NMR}}=6,770 \mathrm{~g} \mathrm{~mol}^{-1}\right.$, $745 \mathrm{mg}, 110 \mu \mathrm{mol})$ with exo-NB-COOH (100 mg, $724 \mu \mathrm{mol})$, EDC (184 mg, $960 \mu \mathrm{mol})$, and DMAP (118 mg, $965 \mu \mathrm{mol})$ in $\mathrm{CH}_{2} \mathrm{Cl}_{2}(7.5 \mathrm{~mL})$. The polymer crude was purified by reprecipitation twice from $\mathrm{CH}_{2} \mathrm{Cl}_{2}$ into cold $\mathrm{MeOH}$ followed by the removal of remaining impurities using preparative SEC (solvent, $\mathrm{CHCl}_{3}$ ) to give $\left.\boldsymbol{s - ( P L L A - N B}\right)_{3}$ as a white solid (489 $\left.\mathrm{mg}\right)$. Yield: $61.9 \%$ $M_{\mathrm{n}, \mathrm{NMR}}=7,910 \mathrm{~g} \mathrm{~mol}^{-1}, M_{\mathrm{n}, \mathrm{SEC}}=9,580 \mathrm{~g} \mathrm{~mol}^{-1}, Ð=1.13$ 
${ }^{1} \mathrm{H}$ NMR (400 MHz, $\left.\mathrm{CDCl}_{3}\right): \delta(\mathrm{ppm}) 7.25$ (s, aromatic), 6.21-6.06 (-CH=CH-in norbornene ring), 5.25-5.04 (m, methine of PLLA backbone, - $\mathrm{CH}_{2} \mathrm{O}-$ ), 3.18-3.05 (m, - $\mathrm{CH}-\mathrm{CH}-\mathrm{CH}_{2} \mathrm{O}-$ in norbornene ring), 2.92 (s, - $\mathrm{CH}-\mathrm{CH}-\mathrm{CH}_{2} \mathrm{O}$ - in norbornene ring), 2.44-2.24 (m, -CHCO- in norbornene ring), 2.101.91 (m, exo-H of - $\mathrm{CH}_{2}$ - in norbornene ring), 1.70-1.48 (m, methyl of PLLA backbone, endo-H of $\mathrm{CH}_{2}$ - in norbornene ring), $1.46-1.31$ (m, bridge head $-\mathrm{CH}_{2}$ - in norbornene ring).

\section{Synthesis of cage $_{3}$-GPLLA}

Method B was used for the cyclopolymerization of $s-(\text { PLLA-NB })_{3}\left(M_{\mathrm{n}, \mathrm{NMR}}=7,910 \mathrm{~g} \mathrm{~mol}^{-1}\right.$, $51.0 \mathrm{mg}, 6.45 \mu \mathrm{mol})$ with $\mathrm{G} 3\left(79.0 \mu \mathrm{L}\right.$ as a $8.0 \mathrm{mmol} \mathrm{L}{ }^{-1}$ solution in dry- $\left.\mathrm{CH}_{2} \mathrm{Cl}_{2}, 0.632 \mu \mathrm{mol}\right)$ in dry$\mathrm{CH}_{2} \mathrm{Cl}_{2}(31.6 \mathrm{~mL})$ for $40 \mathrm{~min}$ to give cage $_{3}$-GPLLA as a pale brown solid (48.9 $\mathrm{mg}$ ). Yield: $95.9 \%$ $M_{\mathrm{n}, \mathrm{SEC}}=51,100 \mathrm{~g} \mathrm{~mol}^{-1}, Ð=1.45$

${ }^{1} \mathrm{H}$ NMR (400 MHz, $\mathrm{CDCl}_{3}$ ): $\delta$ (ppm) 5.69-0.59 (br, alkenyl of poly(norbornene) backbone), 5.27-4.94 (m, methine of PLLA backbone), 1.76-1.37 (m, methyl of PLLA backbone).

\section{Synthesis of $s$-(PLLA-OH) 4}

Method C was used for the polymerization of L-lactide $(1.00 \mathrm{~g}, 8.76 \mathrm{mmol})$ with pentaerythritol $(23.6 \mathrm{mg}, 173 \mu \mathrm{mol})$, and tin(ii)-ethylhexanoate $\left(7.4 \mu \mathrm{L}\right.$ as $0.1 \mathrm{~mol} \mathrm{~L}^{-1}$ solution in toluene, $0.74 \mu \mathrm{mol})$ at $120{ }^{\circ} \mathrm{C}$ for $10 \mathrm{~h}$ to give $\boldsymbol{s}$-(PLLA-OH) 4 as a white solid (754 $\mathrm{mg}$ ). Yield: $73.7 \%$ $M_{\mathrm{n}, \mathrm{NMR}}=5,500 \mathrm{~g} \mathrm{~mol}^{-1}, M_{\mathrm{n}, \mathrm{SEC}}=8,800 \mathrm{~g} \mathrm{~mol}^{-1}, Ð=1.13$ 
${ }^{1} \mathrm{H}$ NMR (400 MHz, $\mathrm{CDCl}_{3}$ ): $\delta$ (ppm) 5.32-5.00 (m, methine of PLLA backbone), 4.46-4.28 (m, $\left.\mathrm{CH}\left(\mathrm{CH}_{3}\right) \mathrm{OH}\right), 4.27-3.99$ (m, - $\left.\mathrm{CH}_{2} \mathrm{O}-\right)$, 2.67 (s,-OH), 1.83-1.37 (m, methyl of PLLA backbone).

\section{Synthesis of $s-(\text { PLLA-NB })_{4}$}

Method A was used for the condensation reaction of $s-(\mathrm{PLLA}-\mathrm{OH})_{4}\left(M_{\mathrm{n}, \mathrm{NMR}}=5,500 \mathrm{~g} \mathrm{~mol}^{-1}\right.$, $707 \mathrm{mg}, 129 \mu \mathrm{mol})$ with exo-NB-COOH (141 mg, $1.02 \mathrm{mmol}), \mathrm{EDC}(296 \mathrm{mg}, 1.54 \mathrm{mmol})$, and DMAP (177 mg, $1.45 \mathrm{mmol})$ in $\mathrm{CH}_{2} \mathrm{Cl}_{2}(7.0 \mathrm{~mL})$. The polymer crude was purified by reprecipitation twice from $\mathrm{CH}_{2} \mathrm{Cl}_{2}$ into cold $\mathrm{MeOH}$ followed by the removal of remaining impurities using preparative SEC (solvent, $\mathrm{CHCl}_{3}$ ) to give s-(PLLA-NB)4 as a white solid (445 mg). Yield: 57.0\% $M_{\mathrm{n}, \mathrm{NMR}}=6,760 \mathrm{~g} \mathrm{~mol}^{-1}, M_{\mathrm{n}, \mathrm{SEC}}=8,290 \mathrm{~g} \mathrm{~mol}^{-1}, Ð=1.18$

${ }^{1} \mathrm{H}$ NMR (400 MHz, $\left.\mathrm{CDCl}_{3}\right): \delta(\mathrm{ppm})$ 6.23-6.05 (m, -CH=CH- in norbornene ring), 5.27-4.99 (m, methine of PLLA backbone), 4.22-4.01 (m, $-\mathrm{CH}_{2} \mathrm{O}-$ ), 3.24-3.03 (m, $-\mathrm{CHCHCH} \mathrm{H}_{2} \mathrm{O}$ - in norbornene ring), $2.92\left(\mathrm{~s},-\mathrm{CHCH}_{2} \mathrm{CHCO}\right.$ - in norbornene ring), 2.36-2.25 (m, -CHCO- in norbornene ring), 2.07-1.87 (m, exo-H of - $\mathrm{CH}_{2}$ - in norbornene ring), 1.70-1.45 (m, methyl of PLLA backbone, endo-H of - $\mathrm{CH}_{2}$ - in norbornene ring), 1.46-1.31 (m, bridge head $-\mathrm{CH}_{2}$ - in norbornene ring).

\section{Synthesis of $\mathrm{cage}_{4}$-GPLLA}

Method B was used for the cyclopolymerization of $s-(\text { PLLA-NB })_{4}\left(M_{\mathrm{n}, \mathrm{NMR}}=6,760 \mathrm{~g} \mathrm{~mol}^{-1}\right.$, $20.0 \mathrm{mg}, 2.96 \mu \mathrm{mol})$ with $\mathrm{G} 3\left(49.3 \mu \mathrm{L}\right.$ as a $8.0 \mathrm{mmol} \mathrm{L}{ }^{-1}$ solution in dry- $\left.\mathrm{CH}_{2} \mathrm{Cl}_{2}, 0.394 \mu \mathrm{mol}\right)$ in dry- 
$\mathrm{CH}_{2} \mathrm{Cl}_{2}(14.8 \mathrm{~mL})$ for $30 \mathrm{~min}$. The byproducts and metal residue were removed by preparative SEC (solvent, $\mathrm{CHCl}_{3}$ ) to give cage $_{4}$-GPLLA as a pale brown solid $(15.9 \mathrm{mg}$ ). Yield: $79.5 \%$

$M_{\mathrm{n}, \mathrm{SEC}}=22,400 \mathrm{~g} \mathrm{~mol}^{-1}, Ð=1.23$

${ }^{1} \mathrm{H}$ NMR (400 MHz, $\mathrm{CDCl}_{3}$ ): $\delta$ (ppm) 5.49-1.10 (br, alkenyl of poly(norbornene) backbone), 5.28-5.01 (m, methine of PLLA backbone), 4.36-3.96 (br, - $\mathrm{CH}_{2} \mathrm{O}-$ ), 1.77-1.42 (m, methyl of PLLA backbone).

\section{Synthesis of $s$-(PTMC-OH)3}

Method C was used for the polymerization of trimethylene carbonate (1.50 g, $10.4 \mathrm{mmol})$ with 1,3,5-trihydroxymethylbenzene $(41.2 \mathrm{mg}, 245 \mu \mathrm{mol})$, and DPP $(3.1 \mathrm{mg}, 12 \mu \mathrm{mol})$ at $80{ }^{\circ} \mathrm{C}$ for 68 h to give $\boldsymbol{s - ( P T M C - O H ) 3 ~ a s ~ a ~ c o l o r l e s s ~ v i s c o u s ~ l i q u i d ~ ( 1 . 5 4 ~ g ) . ~ Y i e l d : ~ 9 9 . 9 \% ~}$

$M_{\mathrm{n}, \mathrm{NMR}}=5,910 \mathrm{~g} \mathrm{~mol}^{-1}, M_{\mathrm{n}, \mathrm{SEC}}=9,710 \mathrm{~g} \mathrm{~mol}^{-1}, Ð=1.10$

${ }^{1} \mathrm{H}$ NMR (400 MHz, $\left.\mathrm{CDCl}_{3}\right): \delta(\mathrm{ppm}) 7.36$ (s, aromatic), 4.45 (t, $\left.J=5.6 \mathrm{~Hz}, \mathrm{ArCH}_{2} \mathrm{O}-\right), 4.35-4.11$ (m, $-\mathrm{COOCH}_{2} \mathrm{CH}_{2} \mathrm{CH}_{2} \mathrm{O}-$ ), 3.73 (t, $J=6.1 \mathrm{~Hz},-\mathrm{CH}_{2} \mathrm{OH}$ ), 2.25-1.83 (m, $-\mathrm{COOCH}_{2} \mathrm{CH}_{2} \mathrm{CH}_{2} \mathrm{O}-$ ).

\section{Synthesis of $s-($ PTMC-NB) 4}

Method A was used for the condensation reaction of $s-($ PTMC-OH $) 3\left(M_{\mathrm{n}, \mathrm{NMR}}=5,910 \mathrm{~g} \mathrm{~mol}^{-}\right.$

1, $1.03 \mathrm{~g}, 174 \mu \mathrm{mol})$ with exo-NB-COOH (147 mg, $1.06 \mathrm{mmol})$, EDC (321 mg, $1.67 \mathrm{mmol})$, and DMAP (192 mg, $1.57 \mathrm{mmol})$ in $\mathrm{CH}_{2} \mathrm{Cl}_{2}(10.3 \mathrm{~mL})$. The polymer crude was purified by reprecipitation twice 
from $\mathrm{CH}_{2} \mathrm{Cl}_{2}$ into cold $\mathrm{MeOH}$ followed by the removal of remaining impurities using preparative SEC (solvent, $\mathrm{CHCl}_{3}$ ) to give $\left.\boldsymbol{s - ( P T M C - N B}\right)_{3}$ as a pale yellow viscous liquid $(891 \mathrm{mg})$. Yield: $81.0 \%$ $M_{\mathrm{n}, \mathrm{NMR}}=6,900 \mathrm{~g} \mathrm{~mol}^{-1}, M_{\mathrm{n}, \mathrm{SEC}}=9,730 \mathrm{~g} \mathrm{~mol}^{-1}, Ð=1.04$

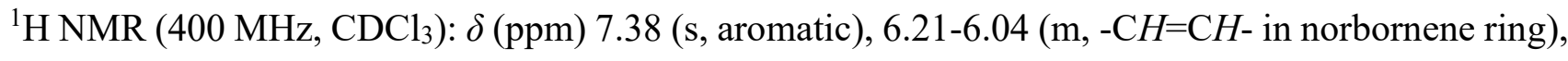
4.37-4.13 (m, - $\mathrm{COOCH}_{2} \mathrm{CH}_{2} \mathrm{CH}_{2} \mathrm{O}-, \mathrm{ArCH}_{2} \mathrm{O}-$ ), 3.03 (m, - $\mathrm{CHCHCH}_{2} \mathrm{O}$ - in norbornene ring), 2.92 (s, $-\mathrm{CHCH}_{2} \mathrm{CHCO}$ - in norbornene ring), 2.31-2.18 (-CHCO- in norbornene ring), 2.16-1.96 (m, $\mathrm{COOCH}_{2} \mathrm{CH}_{2} \mathrm{CH}_{2} \mathrm{O}-$ ), 1.96-1.83 (m, exo-H of - $\mathrm{CH}_{2}$ - in norbornene ring), 1.56-1.46 (m, endo-H of $\mathrm{CH}_{2}$ - in norbornene ring), 1.44-1.31 (m, bridge head $-\mathrm{CH}_{2}$ - in norbornene ring).

\section{Synthesis of cage $_{3}$-GPTMC}

Method B was used for the cyclopolymerization of $s$-(PTMC-NB) $3\left(M_{\mathrm{n}, \mathrm{NMR}}=6,900 \mathrm{~g} \mathrm{~mol}^{-1}\right.$, $49.9 \mathrm{mg}, 7.23 \mu \mathrm{mol})$ with $\mathrm{G} 3\left(106 \mu \mathrm{L}\right.$ as a $8.0 \mathrm{mmol} \mathrm{L}{ }^{-1}$ solution in dry- $\left.\mathrm{CH}_{2} \mathrm{Cl}_{2}, 0.848 \mu \mathrm{mol}\right)$ in dry$\mathrm{CH}_{2} \mathrm{Cl}_{2}(113 \mathrm{~mL})$ for $40 \mathrm{~min}$. The metal residue were removed by preparative SEC (solvent, $\mathrm{CHCl}_{3}$ ) to give cage $_{3}$-GPTMC as a colorless viscous liquid (40.9 mg). Yield: $82.0 \%$ $M_{\mathrm{n}, \mathrm{SEC}}=29,400 \mathrm{~g} \mathrm{~mol}^{-1}, Ð=1.19$

${ }^{1} \mathrm{H}$ NMR (400 MHz, $\mathrm{CDCl}_{3}$ ): $\delta$ (ppm) 7.38 (s, aromatic), 5.51-1.44 (br, alkenyl of poly(norbornene) backbone), 4.50-4.00 (m, - $\mathrm{COOCH}_{2} \mathrm{CH}_{2} \mathrm{CH}_{2} \mathrm{O}-, \mathrm{ArCH}_{2} \mathrm{O}-$ ), 2.27-1.93 (m, $-\mathrm{COOCH}_{2} \mathrm{CH}_{2} \mathrm{CH}_{2} \mathrm{O}-$ ). 


\section{Synthesis of $s$-(PEG-NB $)_{4}$}

Method A was used for the condensation reaction of $s-(\mathrm{PEG}-\mathrm{OH})_{4}\left(M_{\mathrm{n}, \mathrm{GPC}}=5,200 \mathrm{~g} \mathrm{~mol}^{-1}\right.$; $2.00 \mathrm{~g}, 385 \mu \mathrm{mol}$ ) with exo-NB-COOH (426 mg, $3.08 \mathrm{mmol})$, EDC (877 mg, $4.57 \mathrm{mmol})$, and DMAP (553 mg, $4.53 \mathrm{mmol}$ ) in $\mathrm{CH}_{2} \mathrm{Cl}_{2}(20.0 \mathrm{~mL})$. The polymer crude was purified by reprecipitation twice from $\mathrm{CH}_{2} \mathrm{Cl}_{2}$ into cold diethyl ether followed by the removal of remaining impurities using preparative SEC (solvent, $\mathrm{CHCl}_{3}$ ) to give to give $\boldsymbol{s}$-(PEG-NB) 4 as a pale yellow solid (1.06 g). Yield: $45.0 \%$ $M_{\mathrm{n}, \mathrm{NMR}}=5,830 \mathrm{~g} \mathrm{~mol}^{-1}, M_{\mathrm{n}, \mathrm{SEC}}=6,260 \mathrm{~g} \mathrm{~mol}^{-1}, Ð=1.08$ (eluent, DMF; PS std.)

${ }^{1} \mathrm{H}$ NMR (400 MHz, $\mathrm{CDCl}_{3}$ ): $\delta$ (ppm) 6.29-6.01 (m, -CH=CH- in norbornene ring), 4.38-4.17 (m, $\left.\mathrm{CH}_{2} \mathrm{OCO}-\right)$, 3.98-3.31 (m, $\left.\mathrm{C}\left(\mathrm{CH}_{2} \mathrm{O}-\right)_{4},-\mathrm{CH}_{2} \mathrm{CH}_{2} \mathrm{O}-,-\mathrm{CH}_{2} \mathrm{CH}_{2} \mathrm{O}-\right)$, 3.05 (s, -CHCHCO- in norbornene ring), 2.92 (s, - $\mathrm{CHCH}_{2} \mathrm{CHCO}$ - in norbornene ring), 2.36-2.18 (m, -CHCO- in norbornene ring), 2.041.85 (m, exo-H of - $\mathrm{CH}_{2}$ - in norbornene ring), 1.46-1.60 (m, endo- $\mathrm{H}$ of $-\mathrm{CH}_{2}$ - in norbornene ring), 1.281.45 (m, bridge head $-\mathrm{CH}_{2}$ - in norbornene ring).

\section{Synthesis of cage $_{4}$-GPEG}

Method B was used for the cyclopolymerization of $s-(\text { PEG-NB })_{4}\left(M_{\mathrm{n}, \mathrm{NMR}}=5,830 \mathrm{~g} \mathrm{~mol}^{-1}\right.$, $30.0 \mathrm{mg}, 5.15 \mu \mathrm{mol})$ with $\mathrm{G} 3\left(85.8 \mu \mathrm{L}\right.$ as a $8.0 \mathrm{mM}$ solution in dry- $\left.\mathrm{CH}_{2} \mathrm{Cl}_{2}, 0.686 \mu \mathrm{mol}\right)$ in dry- $\mathrm{CH}_{2} \mathrm{Cl}_{2}$ (51.5 mL) for $90 \mathrm{~min}$ to give cage $_{4}$-GPEG as a pale brown solid (28.5 mg). Yield: $93.1 \%$ $M_{\mathrm{n}, \mathrm{SEC}}=16,600 \mathrm{~g} \mathrm{~mol}^{-1}, Ð=1.45$ (eluent, DMF; PS std.)

${ }^{1} \mathrm{H}$ NMR (400 MHz, $\mathrm{CDCl}_{3}$ ): $\delta$ (ppm) 5.65-1.09 (br, alkenyl of poly(norbornene) backbone), 3.86-3.29 $\left(\mathrm{C}\left(\mathrm{CH}_{2} \mathrm{O}-\right)_{4},-\mathrm{CH}_{2} \mathrm{CH}_{2} \mathrm{O}-,-\mathrm{CH}_{2} \mathrm{CH}_{2} \mathrm{O}-\right)$. 
Table S1. Molecular characterization of cage-GPs with diverse polymer backbones $^{a}$

\begin{tabular}{|c|c|c|c|c|c|c|c|}
\hline sample & MM & {$[\mathrm{MM}]_{0} /[\mathrm{G} 3]_{0}$} & {$[\mathrm{MM}]_{0}$} & $\begin{array}{l}\text { time } \\
\text { (min) }\end{array}$ & $M_{\mathrm{n}, \mathrm{SEC}}{ }^{b}$ & $\bigoplus^{b}$ & $\begin{array}{l}\text { yield } \\
(\%)\end{array}$ \\
\hline cage $_{3}$-GPLLA & $\begin{array}{c}s-(\mathrm{PLLA}-\mathrm{NB})_{3} \\
\left(M_{\mathrm{n}, \mathrm{NMR}}=7,910, M_{\mathrm{n}, \mathrm{SEC}}\right. \\
=9,580, \emptyset=1.13)\end{array}$ & $10 / 1$ & 0.2 & 40 & 51,100 & 1.45 & 95.9 \\
\hline cage $_{4}$-GPLLA & $\begin{array}{c}s-(\mathrm{PLLA}-\mathrm{NB})_{4} \\
\left(M_{\mathrm{n}, \mathrm{NMR}}=6,760, M_{\mathrm{n}, \mathrm{SEC}}\right. \\
=8,290, \emptyset=1.18)\end{array}$ & $7.5 / 1$ & 0.2 & 30 & 22,400 & 1.23 & 79.5 \\
\hline cage $_{3}-\mathrm{GPTMC}$ & $\begin{array}{c}s-(\mathrm{PTMC}-\mathrm{NB})_{3} \\
\left(M_{\mathrm{n}, \mathrm{NMR}}=6,900, M_{\mathrm{n}, \mathrm{SEC}}\right. \\
=9,730, \emptyset=1.04)\end{array}$ & $10 / 1$ & 0.075 & 40 & 29,400 & 1.19 & 82.0 \\
\hline cage $_{4}-\mathrm{GPEG}$ & $\begin{array}{c}s-(\mathrm{PEG}-\mathrm{NB})_{4} \\
\left(M_{\mathrm{n}, \mathrm{NMR}}=5,830, M_{\mathrm{n}, \mathrm{SEC}}\right. \\
\left.=6,260,{ }^{c} Đ=1.08^{c}\right)\end{array}$ & $7.5 / 1$ & 0.1 & 90 & 16,600 & 1.45 & 93.1 \\
\hline
\end{tabular}

${ }^{a}$ Polymerization condition: Temperature, r.t.; Atmosphere, Ar. ${ }^{b}$ Determined by SEC in THF using PSt as standards. ${ }^{c}$ Determined by SEC in DMF using PSt as standards. 


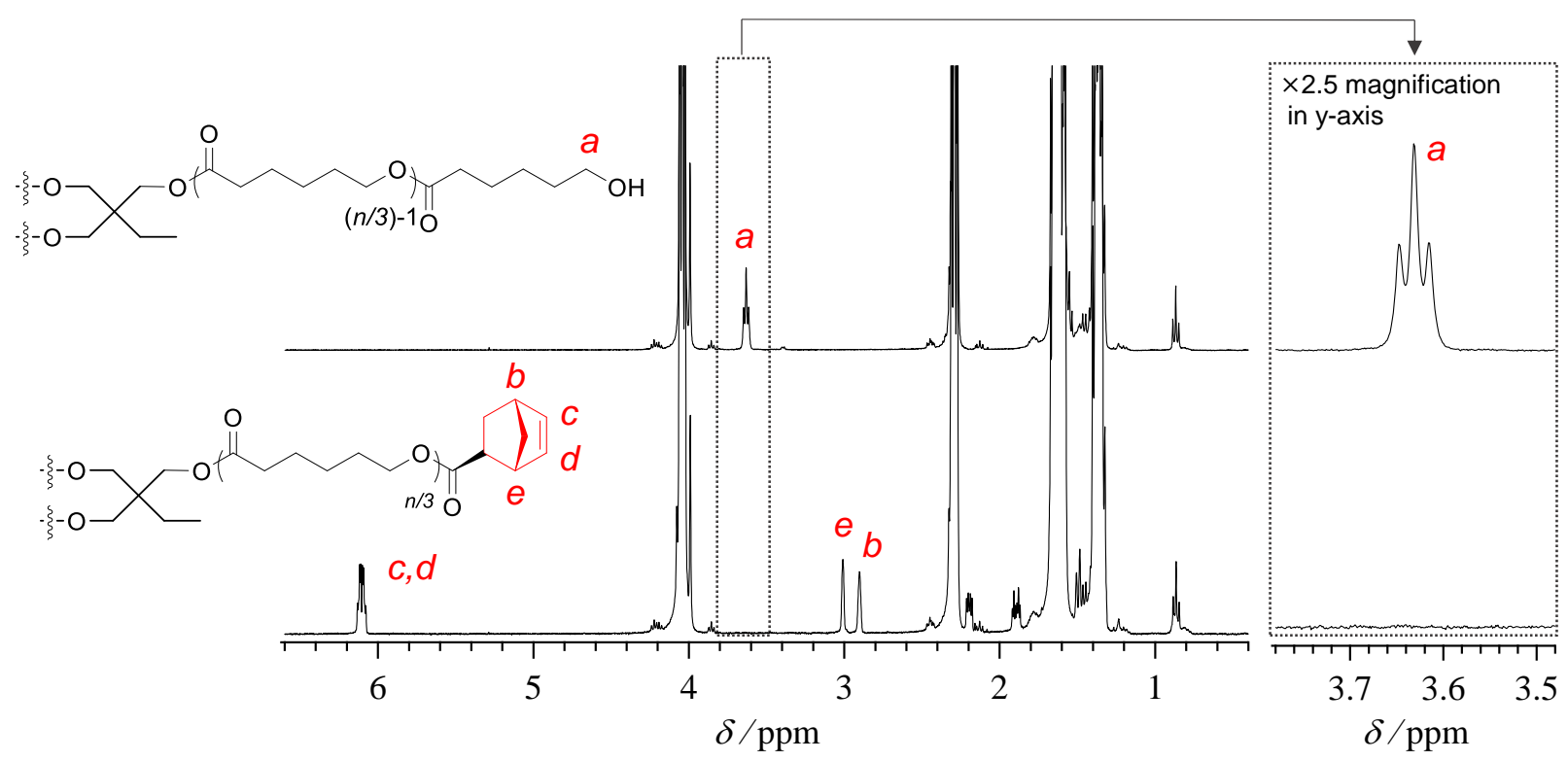

Figure S1. ${ }^{1} \mathrm{H}$ NMR spectra of $s-(\mathrm{PCL}-\mathrm{OH})_{3}$ (upper) and $s$-(PCL-NB) $)_{3}$ (lower) in $\mathrm{CDCl}_{3}$.

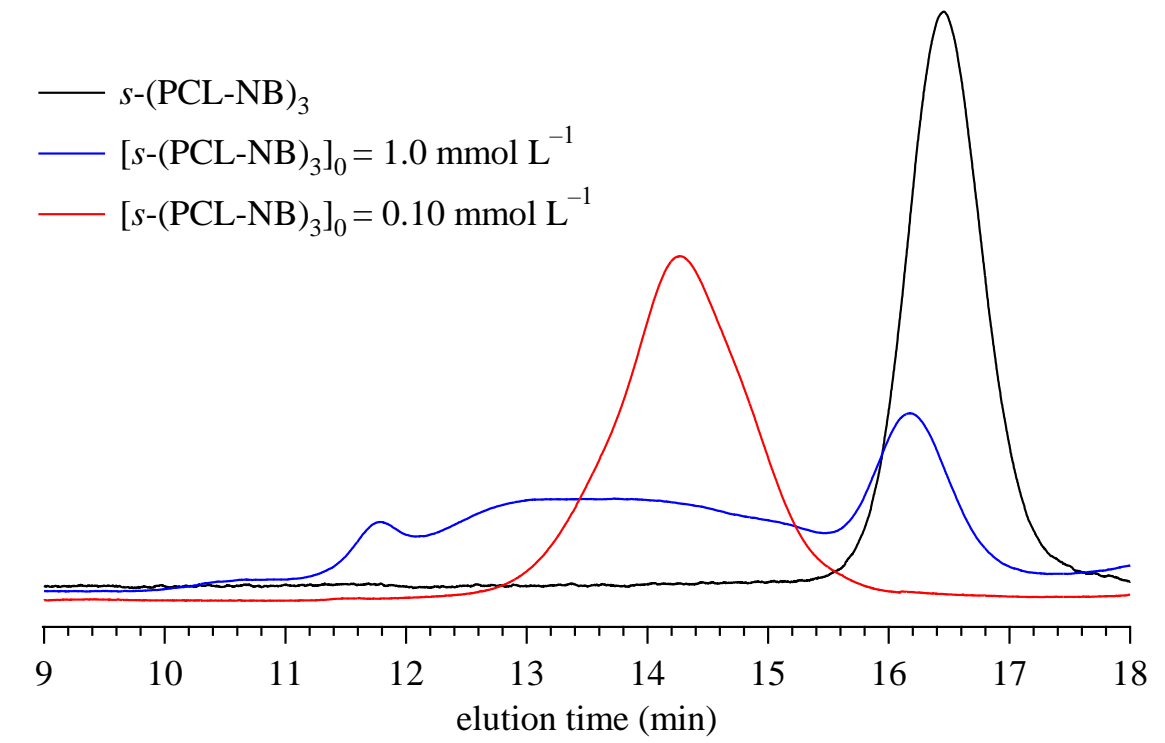

Figure S2. SEC traces of three-armed star-shaped macromonomer ( $s$-(PCL-NB)3, red) and cyclopolymerized products under various macromonomer concentration $\left(\left[\boldsymbol{s}-(\mathbf{P C L}-\mathbf{N B})_{3}\right]_{0}\right)$ with 1.0 (blue) and $0.10 \mathrm{mmol} \mathrm{L}^{-1}$ (red) (RI detection; eluent, THF). 
Chart S1. Expected chemical structures of multiple G3 adducts
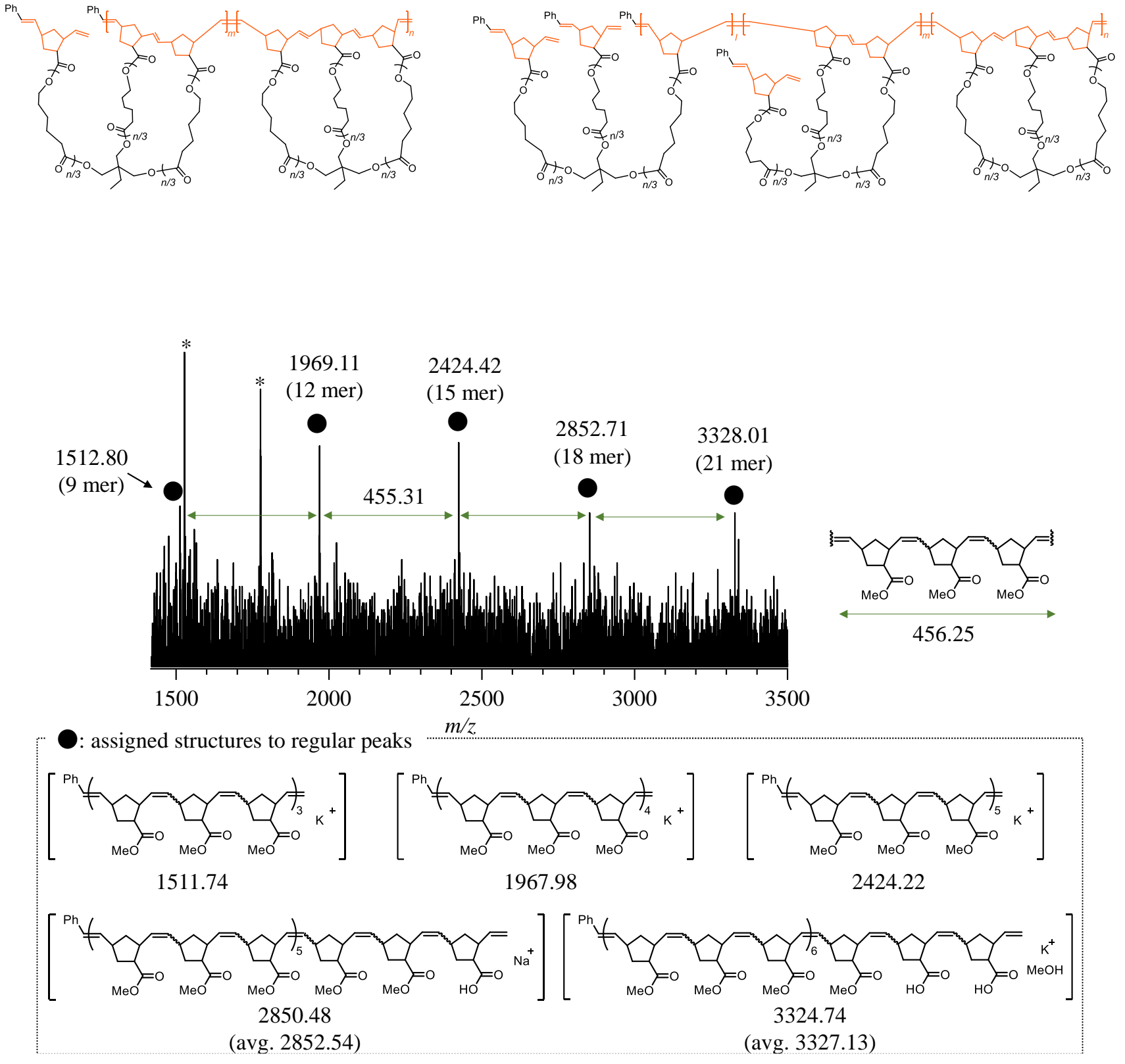

Figure S3. MALDI-TOF MS analysis of methanolysis product of cage 3 -GPCL $\left(M_{\mathrm{n}, \mathrm{SEC}}=28,600 \mathrm{~g}\right.$ $\mathrm{mol}^{-1}, Ð=1.16$; prepared by the cyclopolymerization with $\left[s-(\mathrm{PCL}-\mathrm{NB})_{3}\right]_{0} /[\mathrm{G} 3]_{0}$ ratio of 10$)$. The peaks marked by asterisk were not assignable to any possible methanolysis products. Note that average calculated mass (denoted as avg.) was shown below the expected chemical structures because their monoisotopic signals were hardly seen. 


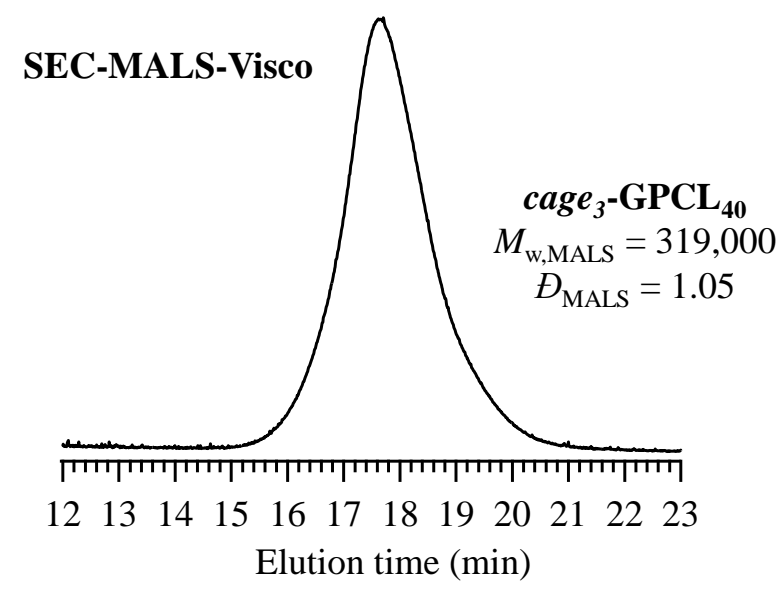

Figure S4. SEC trace of cage $_{3}$-GPCL 40 obtained from SEC with higher exclusion limit (RI detection, eluent; THF). 
(a)
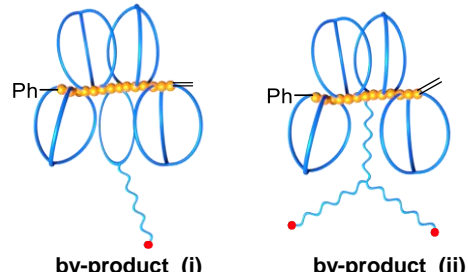

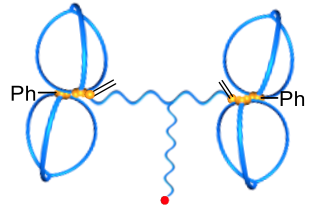

by-product (iii)

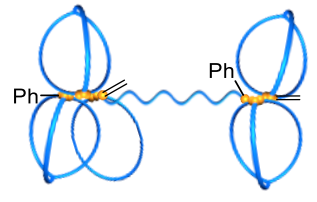

by-product (iv)

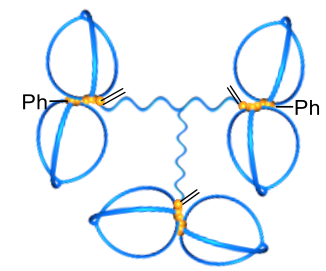

by-product (v)

(b)

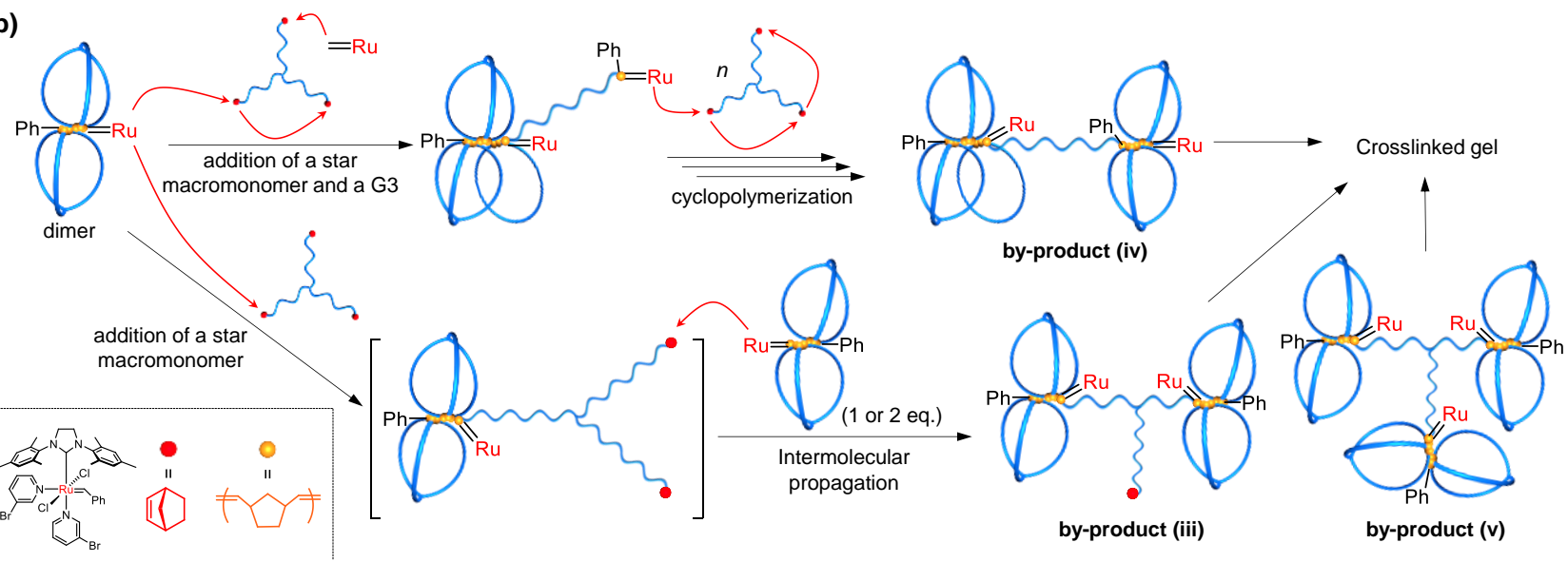

(c)

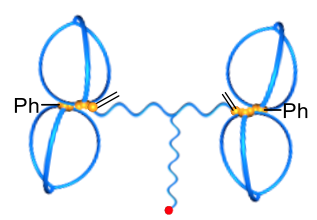

by-product (iii)

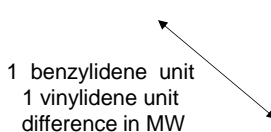

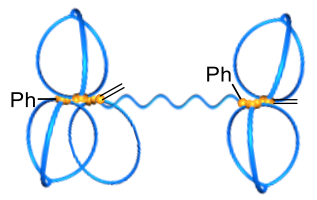

by-product (iv)

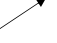

1 benzylidene unit 1 vinylidene unit difference in MW

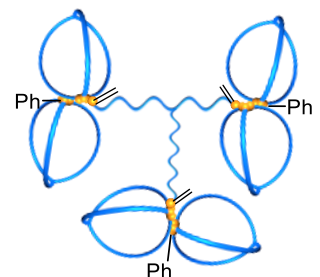

by-product (v)

4 2 benzylidene units 2 vinylidene unit

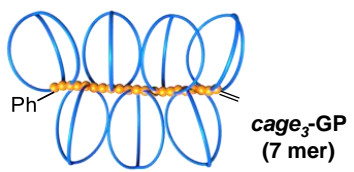

Figure S5. Possible by-product formations and their discrimination from the desired cage-GPs. (a) Various kinds of by-products possibly formed during the cyclopolymerization of $s$-(PCL-NB)3. (b) Possible pathways to form the by-products (iii), (iv), and (v). (c) Structural comparisons between the by-products and desired cage $_{3}$-GPs with different number of cage units, in which the by-products show molecular-weights difference from that of cage-GPs due to presence of extra benzylidene groups $(=\mathrm{CHPh})$ and vinylidene groups $\left(=\mathrm{CH}_{2}\right)$ by the multiple $\mathrm{G} 3$ addition. Note that the structures shown in Figure S4c are the final products obtained after quenching with ethyl vinyl ether. 


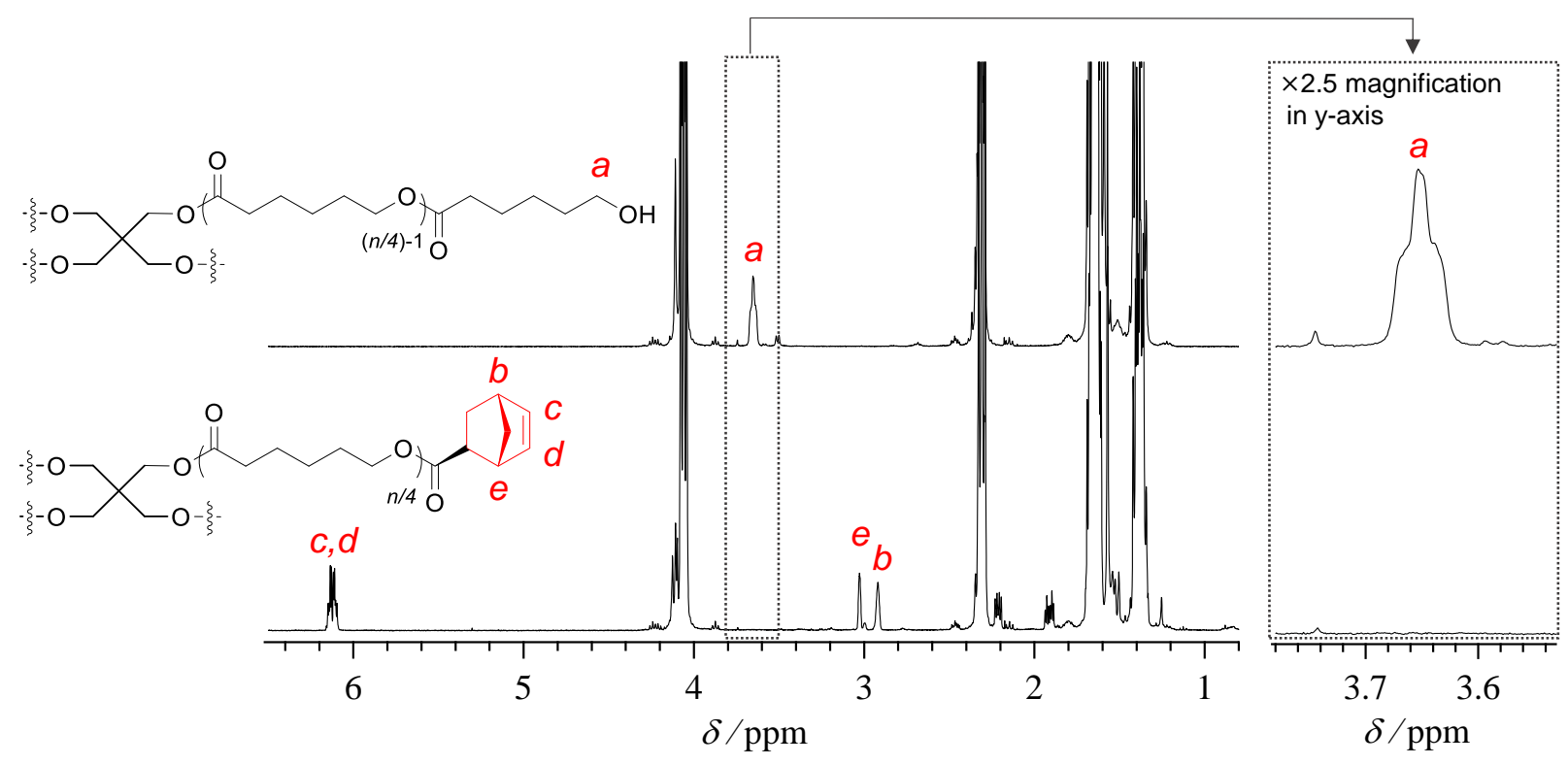

Figure S6. ${ }^{1} \mathrm{H}$ NMR spectra of $s-(\mathrm{PCL}-\mathrm{OH})_{4}$ (upper) and $s-(\mathrm{PCL}-\mathrm{NB})_{4}$ (lower) in $\mathrm{CDCl}_{3}$.

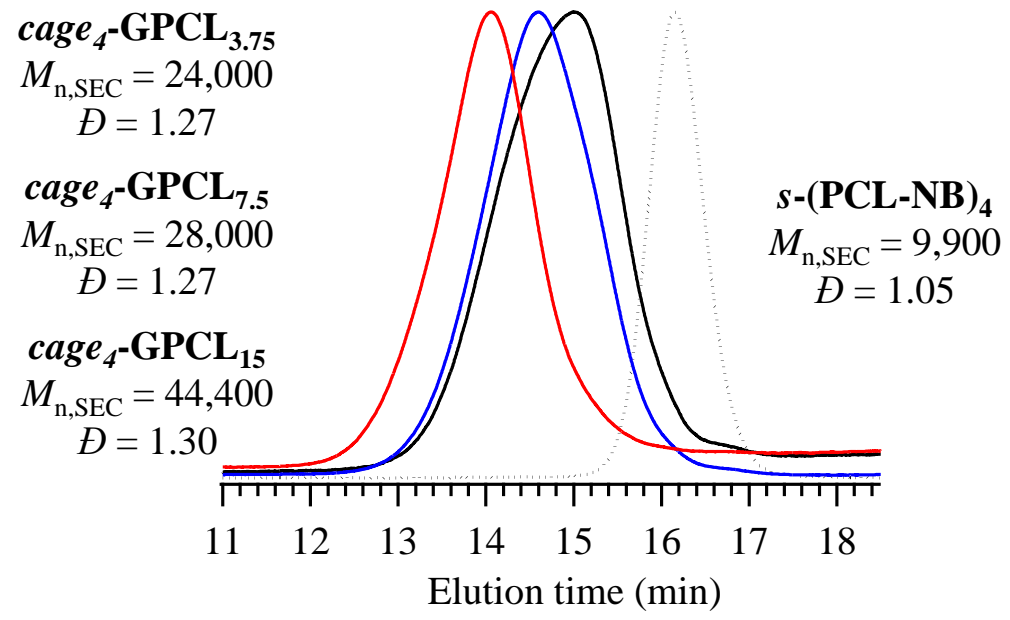

Figure S7. SEC traces of $s$-(PCL-NB)4 (dotted line) and cage $\boldsymbol{e}_{4}$-GPCLs with different molecular weight (black, blue, and red). 


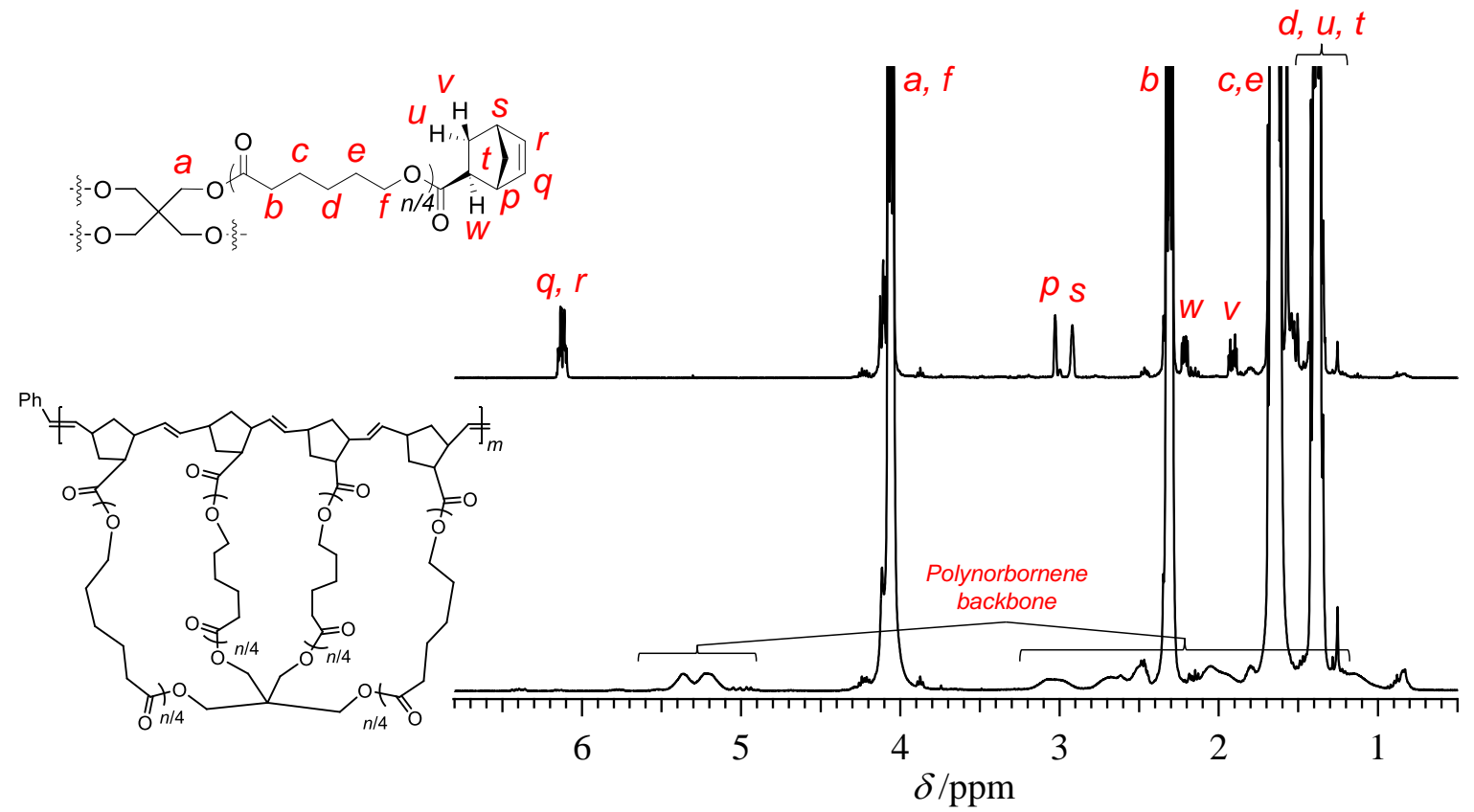

Figure S8. ${ }^{1} \mathrm{H}$ NMR spectra of $\boldsymbol{s}$-(PCL-NB)4 (upper) and cage $_{4}-\mathbf{G P C L}_{3.75}$ (lower) in $\mathrm{CDCl}_{3}$ (400 $\mathrm{MHz}$ ).

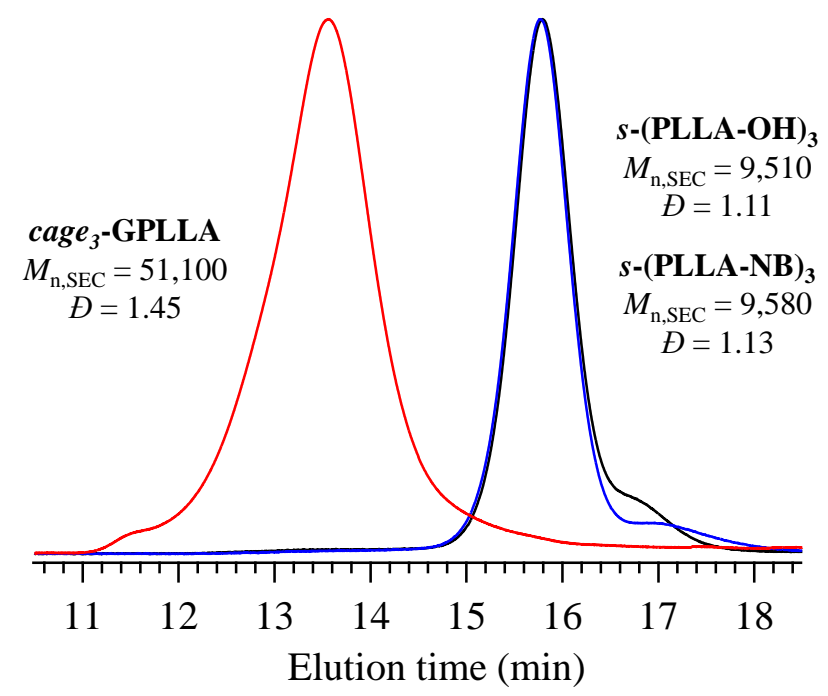

Figure S9. SEC traces of $\boldsymbol{s}$-(PLLA-OH) 3 (black), $\boldsymbol{s}$-(PLLA-NB) 3 (blue), and cage $_{3}$-GPLLA (red) in THF. 


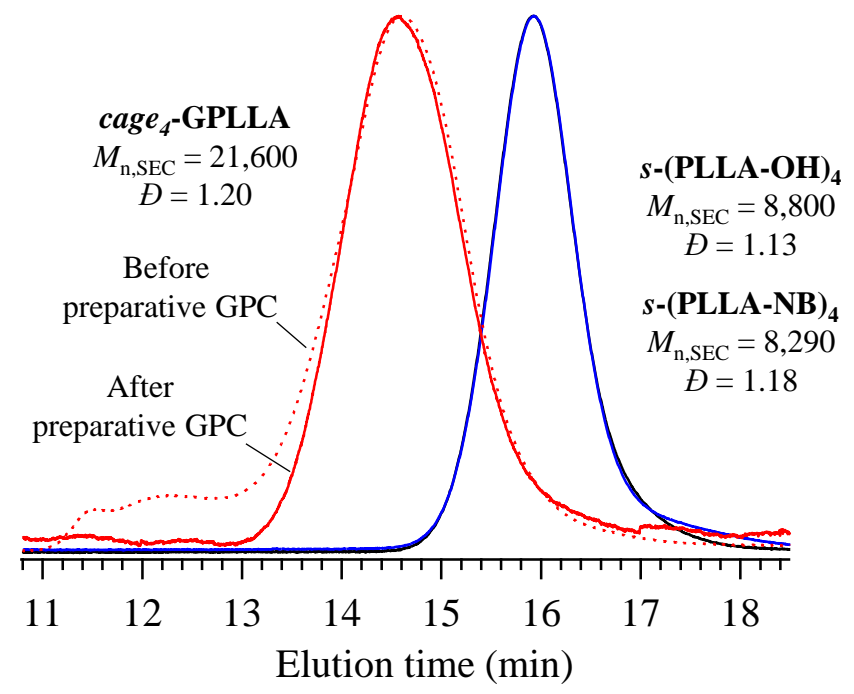

Figure S10. SEC traces of $s$-(PLLA-OH)4 (black), $s$-(PLLA-NB) 4 (blue), and cage $_{4}$-GPLLA (dotted line, before purification; red solid line, after the purification using preparative SEC) in THF.

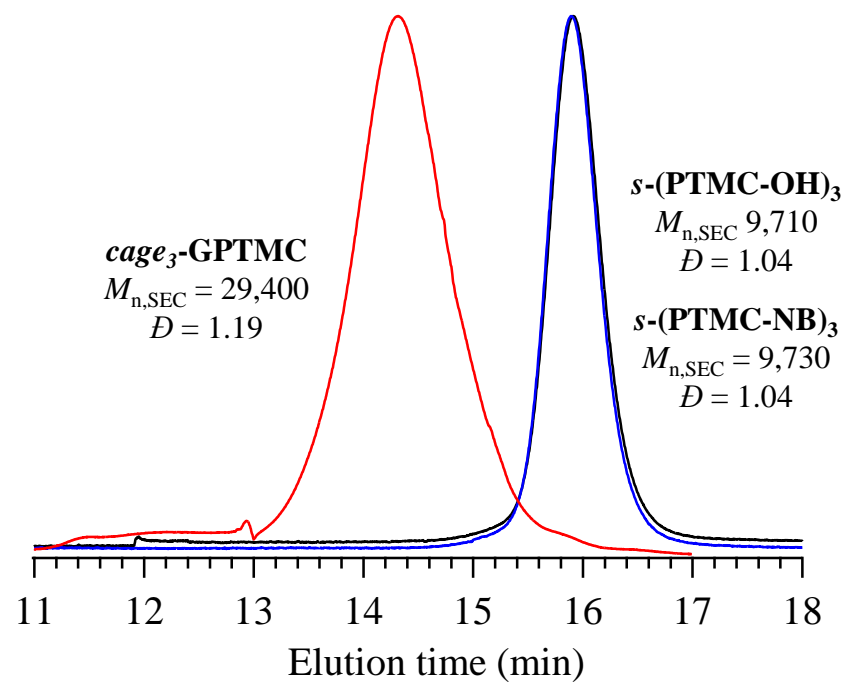

Figure S11. SEC traces of $\boldsymbol{s}-\left(\right.$ PTMC-OH)3 (black), $\boldsymbol{s}$-(PTMC-NB)3 (blue), and cage $_{3}$-GPTMC (red) in THF. 


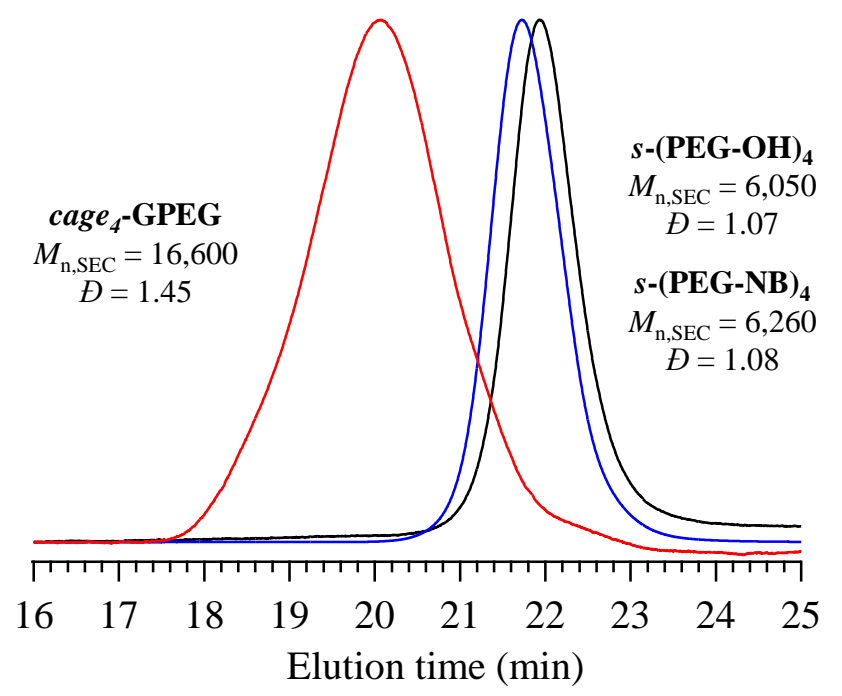

Figure S12. SEC traces of $\boldsymbol{s}$-(PEG-OH)4 (black), $\boldsymbol{s}$-(PEG-NB) 4 (blue), and cage $_{4}$-GPEG (red) in DMF.
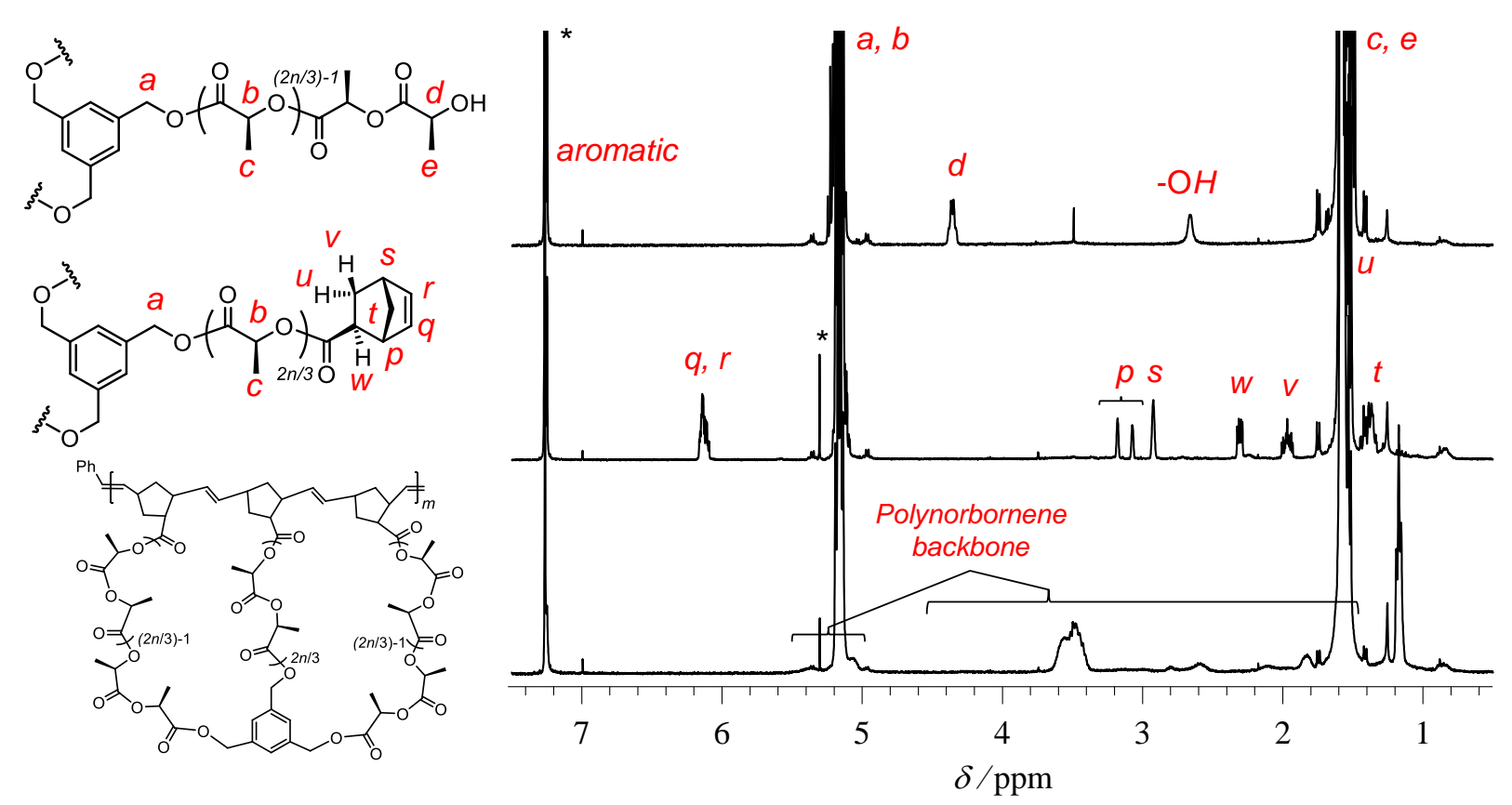

Figure S13. ${ }^{1} \mathrm{H}$ NMR spectra of $\boldsymbol{s}$-(PLLA-OH)3 (upper), $\boldsymbol{s}$-(PLLA-NB)3 (middle), and cage $_{3}$-GPLLA (lower) in $\mathrm{CDCl}_{3}(400 \mathrm{MHz})$. 


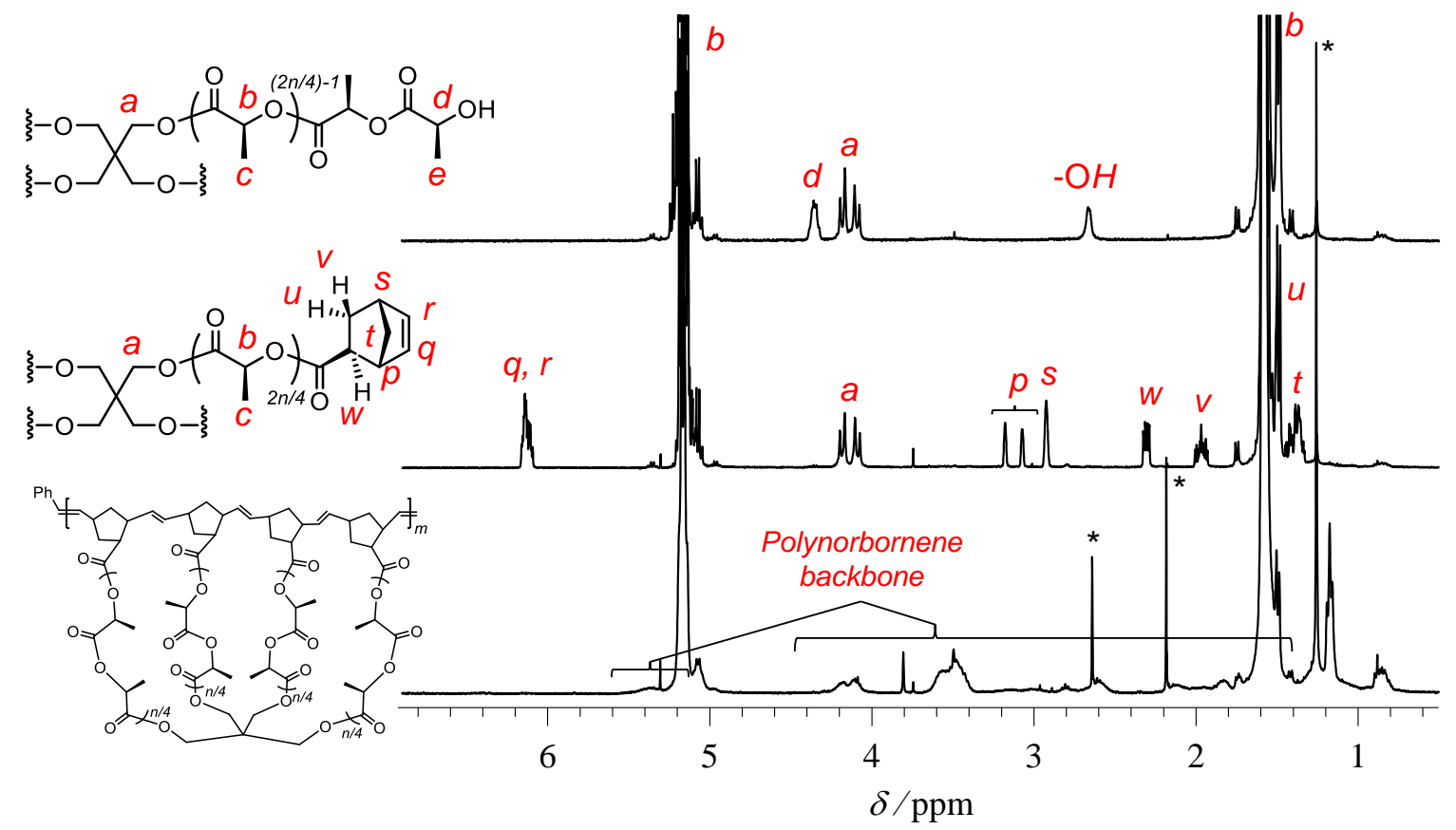

Figure S14. ${ }^{1} \mathrm{H}$ NMR spectra of $s$-(PLLA-OH)4 (upper), $s$-(PLLA-NB) 4 (middle), and cage $_{4}$-GPLLA (lower) in $\mathrm{CDCl}_{3}(400 \mathrm{MHz})$.
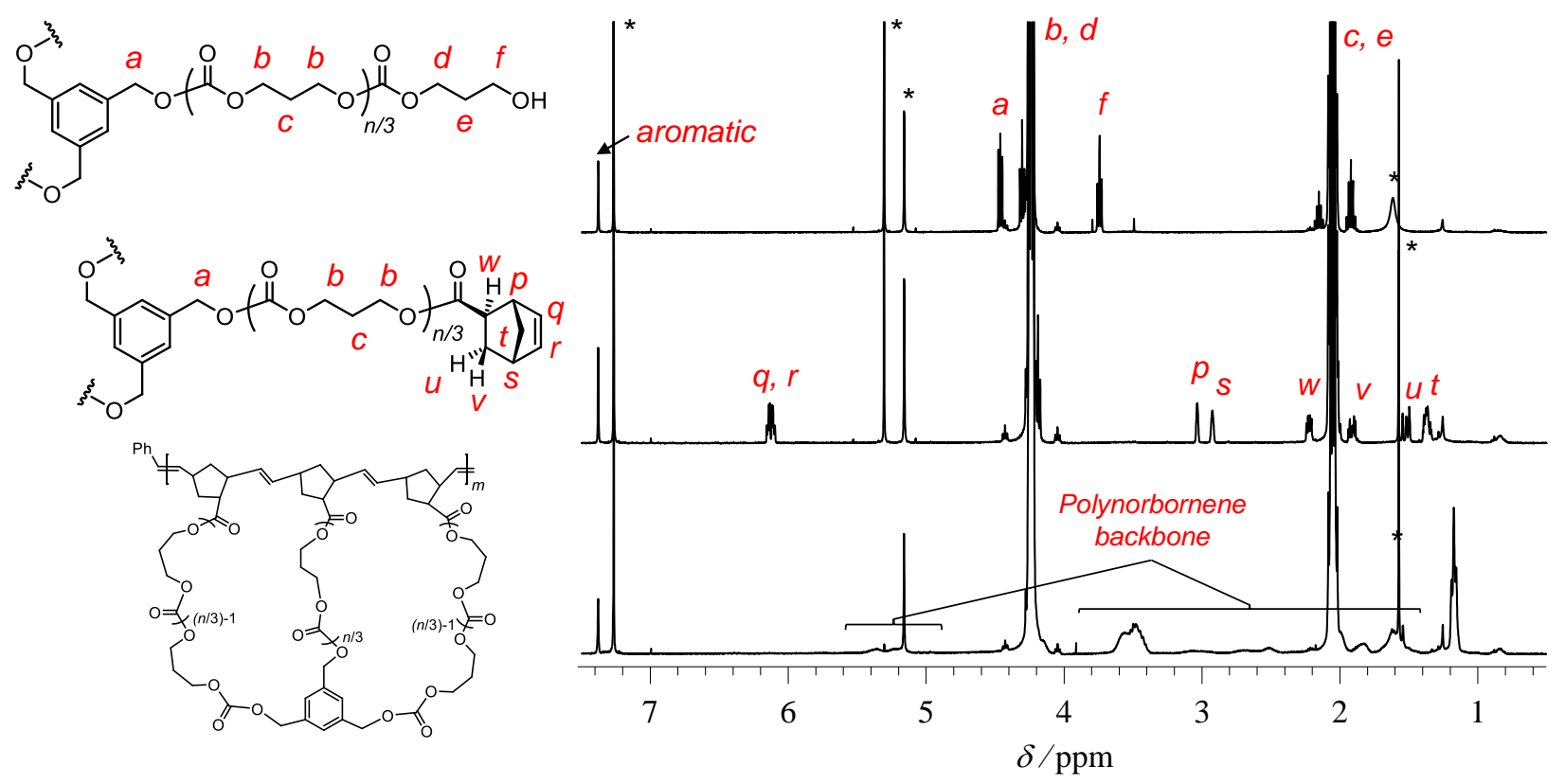

Figure S15. ${ }^{1} \mathrm{H}$ NMR spectra of $s$-(PTMC-OH)3 (upper), s-(PTMC-NB)3 (middle), and cage $_{3}$ GPTMC (lower) in $\mathrm{CDCl}_{3}(400 \mathrm{MHz})$. 


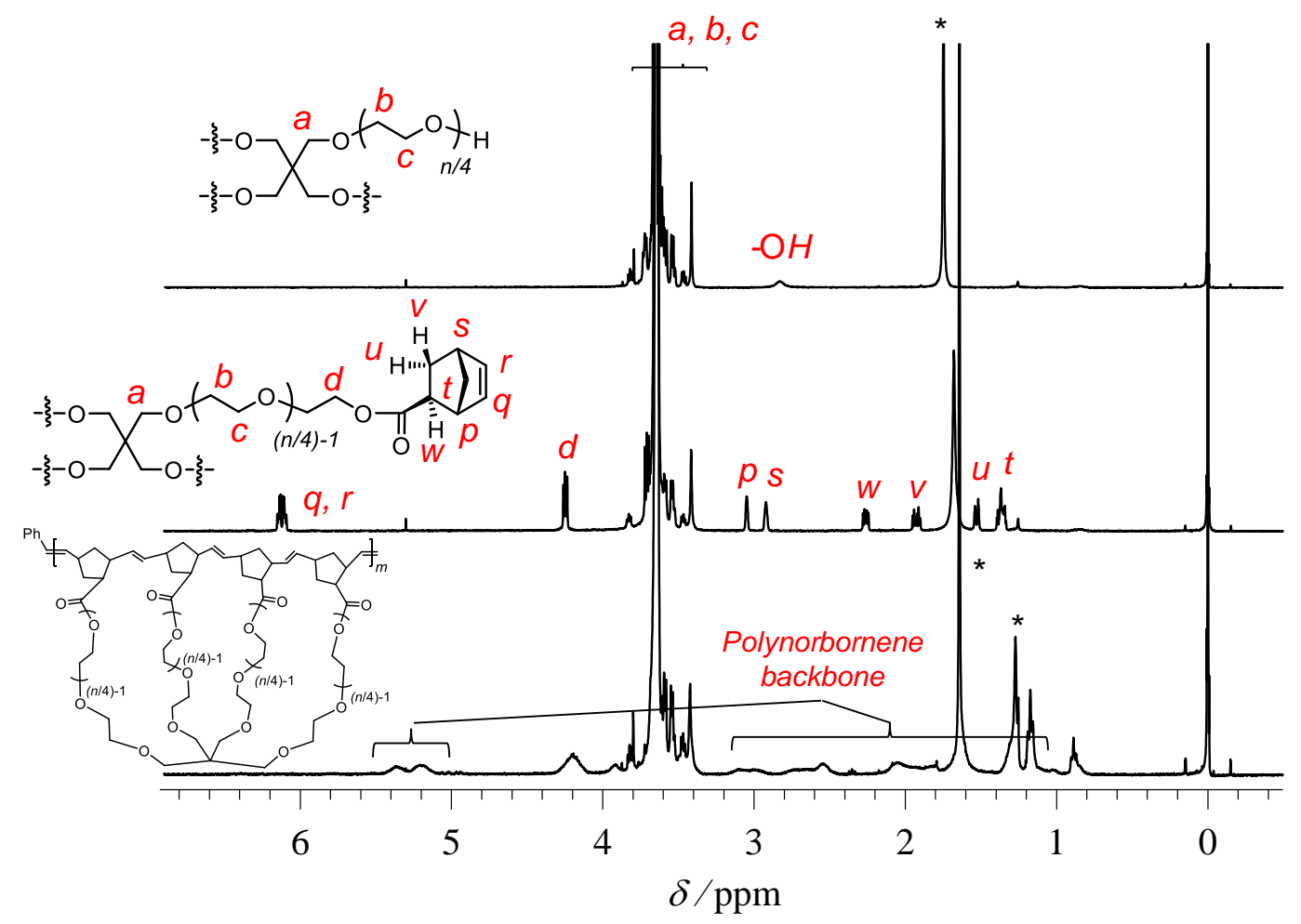

Figure S16. ${ }^{1} \mathrm{H}$ NMR spectra of $\boldsymbol{s}$-(PEG-OH)4 (upper), $\boldsymbol{s}$-(PEG-NB)4 (middle), and cage $_{4}$-GPEG (lower) in $\mathrm{CDCl}_{3}(400 \mathrm{MHz})$.

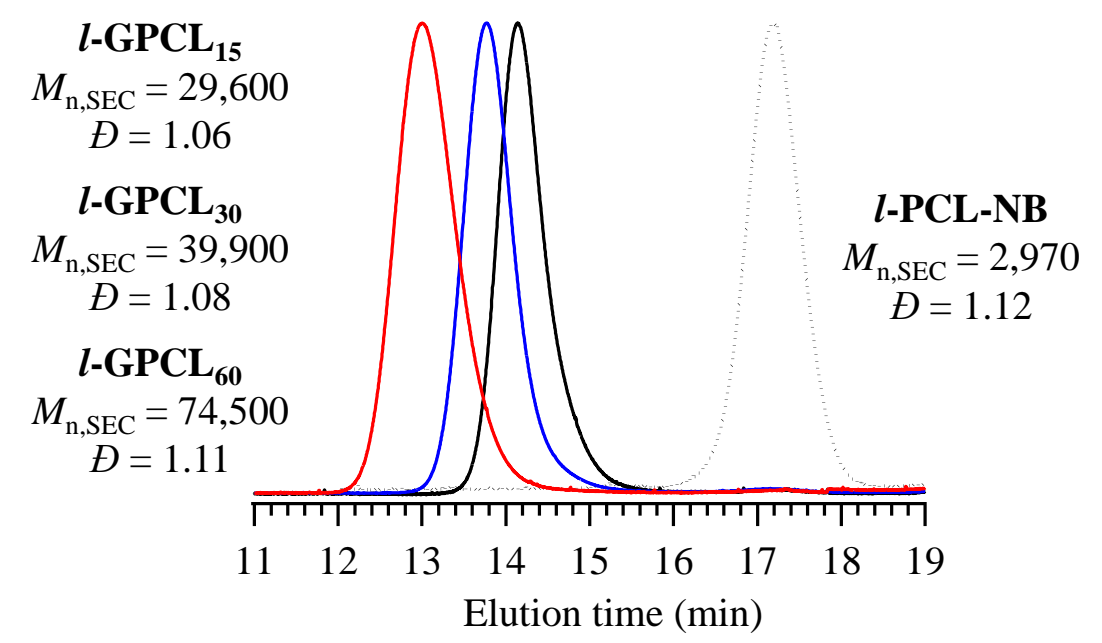

Figure S17. SEC traces of $\boldsymbol{l}$-PCL-NB (dotted line) and $\boldsymbol{l}$-GPCLs with different molecular weight (black, blue, and red). 


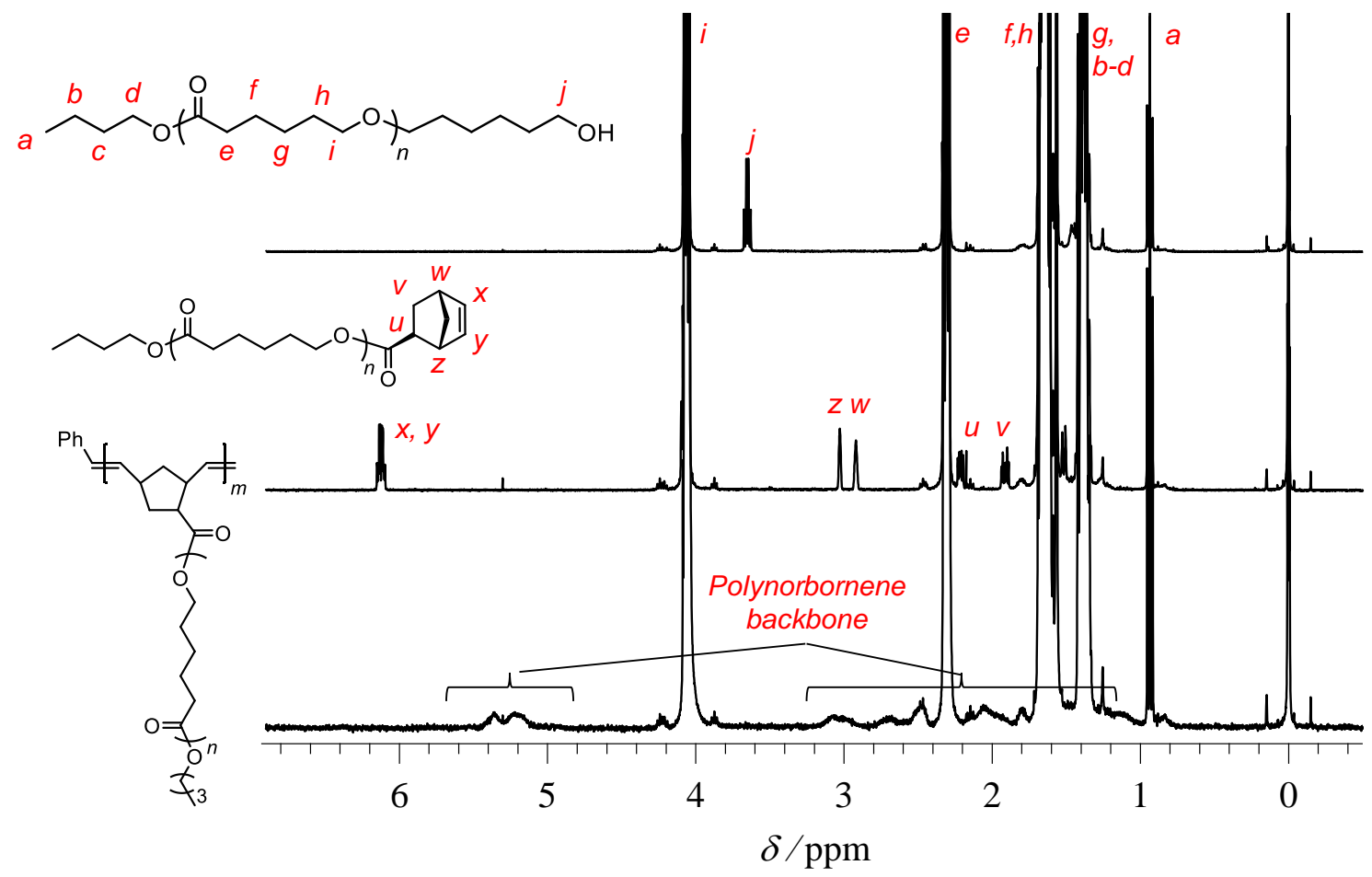

Figure S18. ${ }^{1} \mathrm{H}$ NMR spectra of $\boldsymbol{l}$-PCL-OH (upper), $\boldsymbol{l}$-PCL-NB (middle), and $\boldsymbol{l}$-GPCL (lower) in $\mathrm{CDCl}_{3}(400 \mathrm{MHz})$.

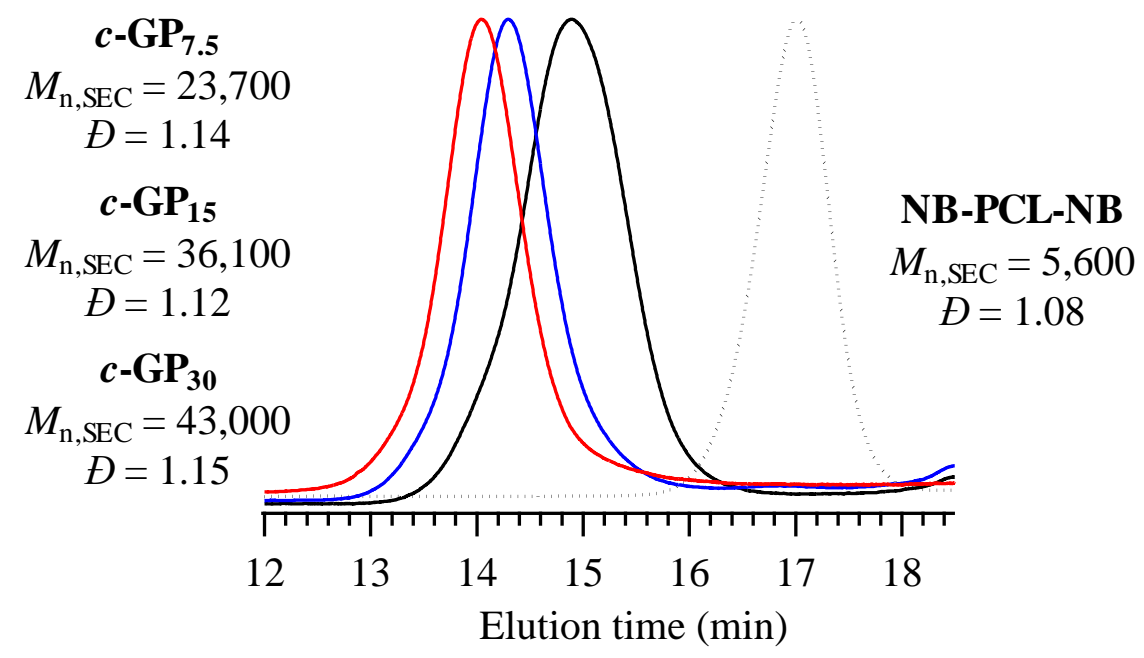

Figure S19. SEC traces of NB-PCL-NB (dotted line) and $\boldsymbol{c}$-GPCLs with different molecular weight (black, blue, and red). 


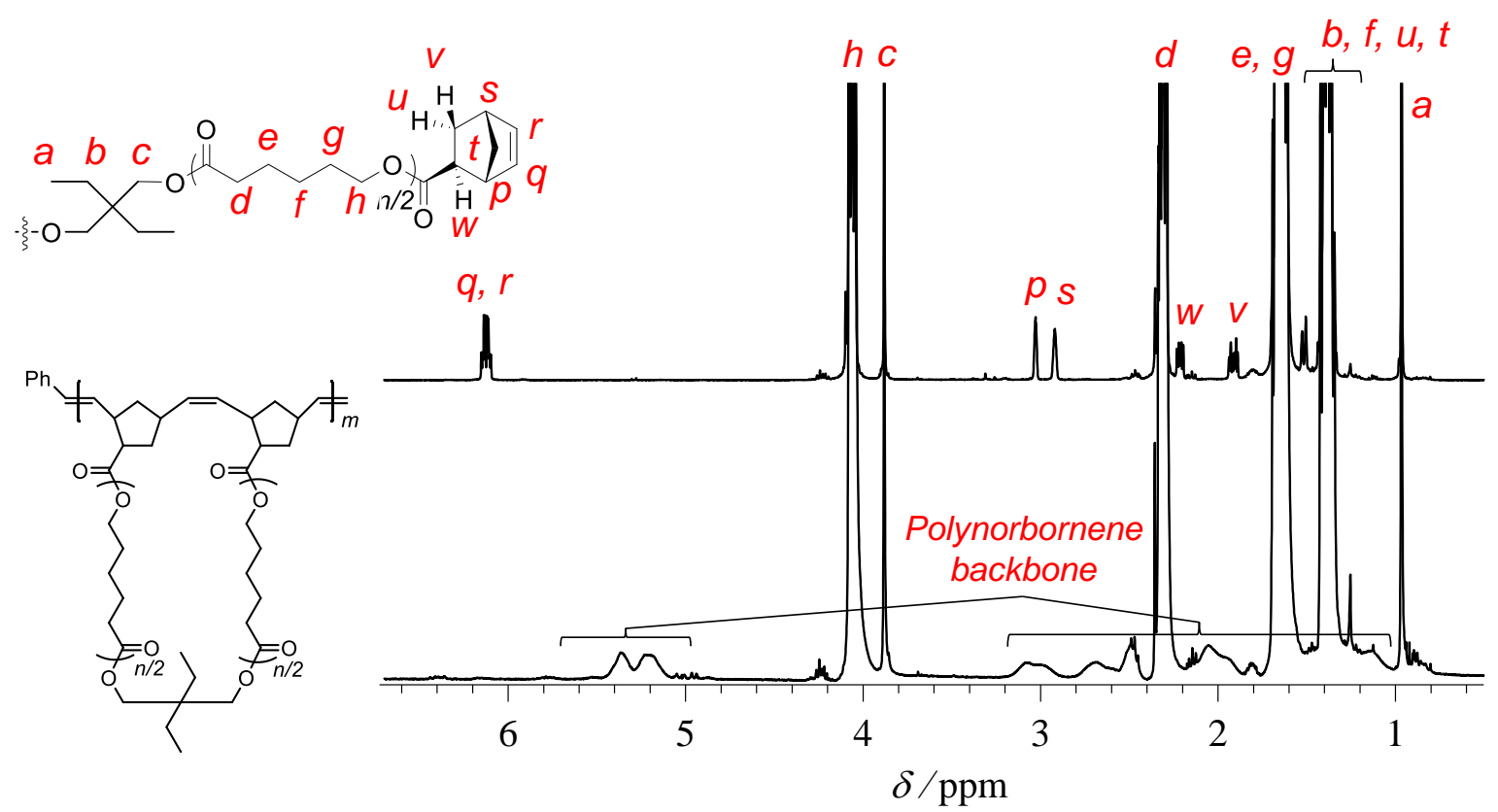

Figure S20. ${ }^{1} \mathrm{H}$ NMR spectra of HO-PCL-OH (upper), NB-PCL-NB (middle), and $\boldsymbol{c}$-GPCL (lower) in $\mathrm{CDCl}_{3}(400 \mathrm{MHz})$.

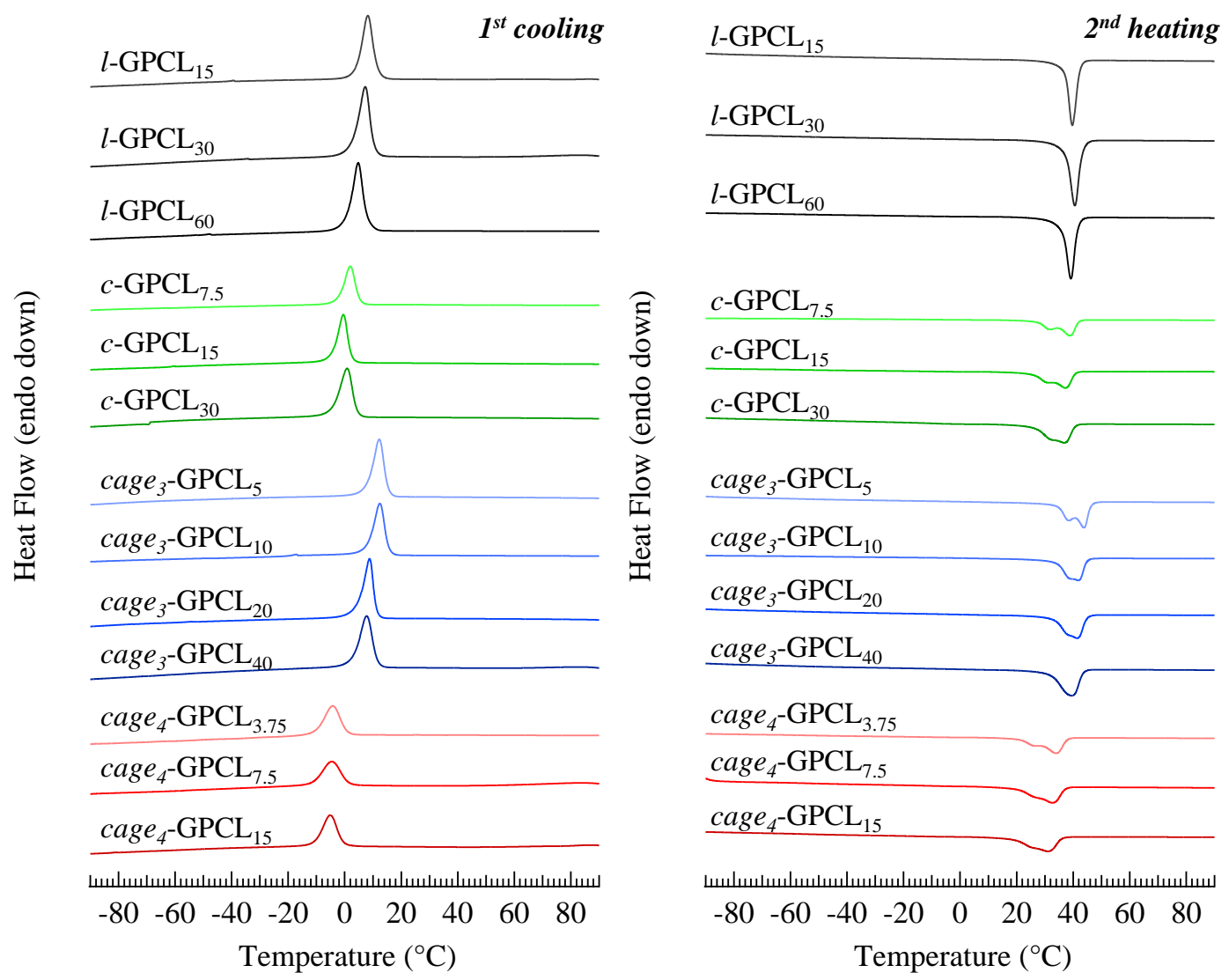

Figure S21. DSC thermograms during $1^{\text {st }}$ cooling (left) and $2^{\text {nd }}$ heating (right) of topological GPCLs $\left(\mathrm{N}_{2}\right.$ flow, heating and cooling rates: 10 and $20{ }^{\circ} \mathrm{C} \mathrm{min}{ }^{-1}$, respectively). 
Table S2. WAXD and SAXS analysis on topological GPCLs and their monomeric counterparts ${ }^{a}$

\begin{tabular}{cccccccc}
\hline sample & $\begin{array}{c}M_{\mathrm{n}, \mathrm{MALS}^{b}} \\
\left(\mathrm{~g} \mathrm{~mol}^{-1}\right)\end{array}$ & $\Xi^{c}$ & $\begin{array}{c}X_{\mathrm{WAXD}^{d}} \\
(\%)\end{array}$ & $l_{\mathrm{c}}(\mathrm{nm})^{e}$ & $l_{\mathrm{a}}(\mathrm{nm})^{e}$ & $L_{\mathrm{p}}(\mathrm{nm})^{e}$ & $l_{\mathrm{c}} / L_{\mathrm{p}}(\%)^{e}$ \\
\hline$l$-GPCL & 33,200 & 1.06 & 42.1 & 4.5 & 6.7 & 11.2 & 40.0 \\
$c$-GPCL & 34,300 & 1.14 & 33.7 & 4.5 & 8.0 & 12.5 & 36.0 \\
cage $_{3}$-GPCL & 42,900 & 1.20 & 27.4 & 4.4 & 7.5 & 11.9 & 36.7 \\
cage $_{4}$-GPCL & 40,200 & 1.27 & 21.8 & 4.5 & 9.4 & 13.9 & 32.5 \\
\hline -PCL & 1,610 & 1.12 & 40.3 & 3.2 & 7.8 & 11.0 & 28.9 \\
$c$-PCL & 3,130 & 1.15 & 39.0 & 3.6 & 7.0 & 10.6 & 33.8 \\
cage $_{3}$-PCL & 3,960 & 1.10 & 27.8 & 4.3 & 7.1 & 11.5 & 37.6 \\
cage $_{4}$-PCL & 5,550 & 1.08 & 34.0 & 4.5 & 7.0 & 11.5 & 38.7 \\
\hline
\end{tabular}

${ }^{a}$ Measurement conditions for WAXD and SAXS: temperature: $\mathrm{rt}$, sample-to-detector distance: $2.5 \mathrm{~m}$ (SAXS), blank: Kapton. Each sample was isothermally crystallized at $30{ }^{\circ} \mathrm{C}$ for $48 \mathrm{~h}$ prior to the measurements. ${ }^{b}$ Determined by triple-detection SEC equipped with multiangle light scattering, viscosity, and refractive index detectors (SEC-MALS-Visco) in THF. ${ }^{c}$ Determined by SEC equipped with RI detector in THF. ${ }^{d}$ Crystallinity was determined by the peak separation of WAXD profile. ${ }^{e}$ Determined by the correlation function analysis of SAXS profile.
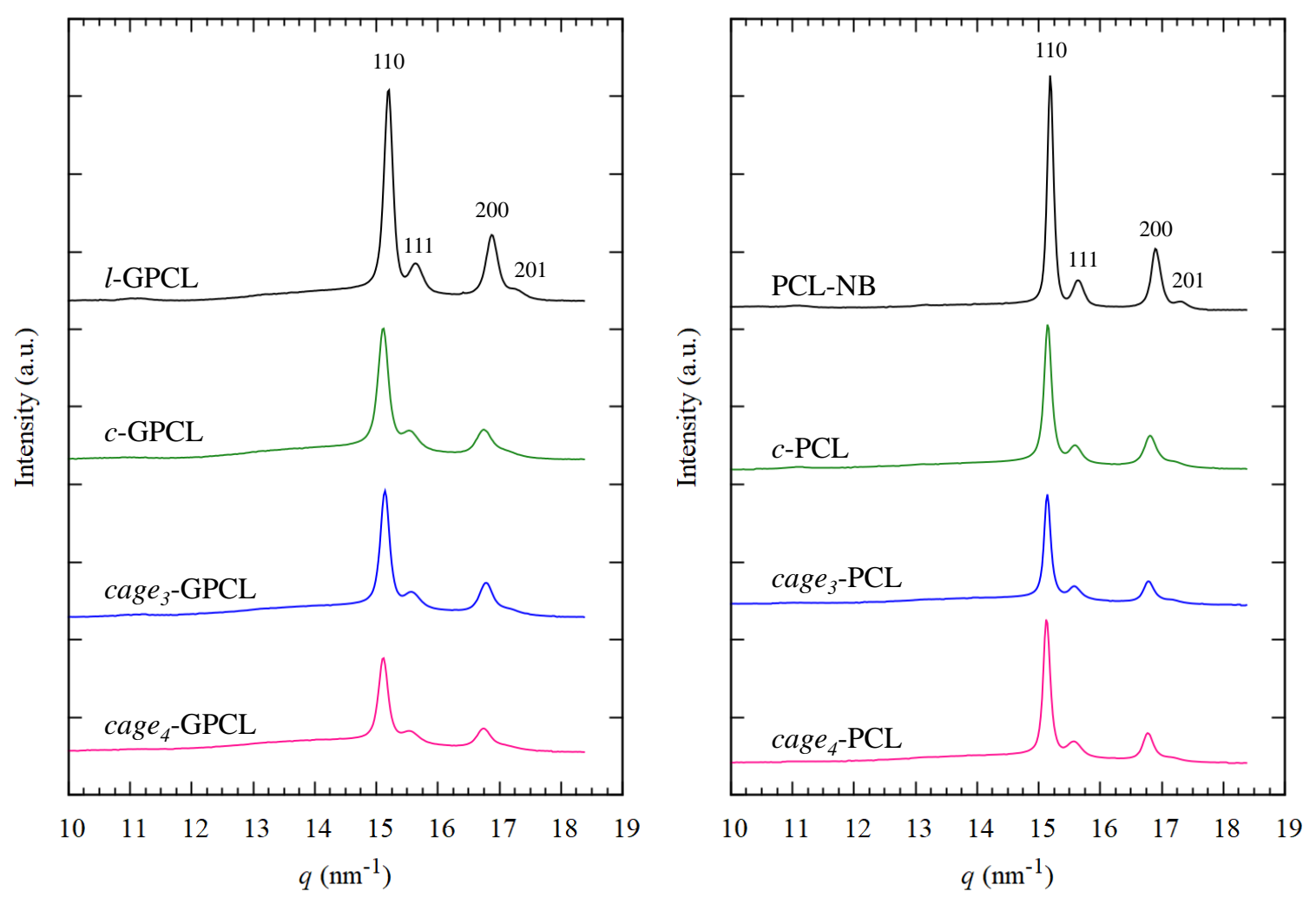

Figure S22. WAXD profiles of topological GPCLs (left) and corresponding monomeric counterparts (right). 

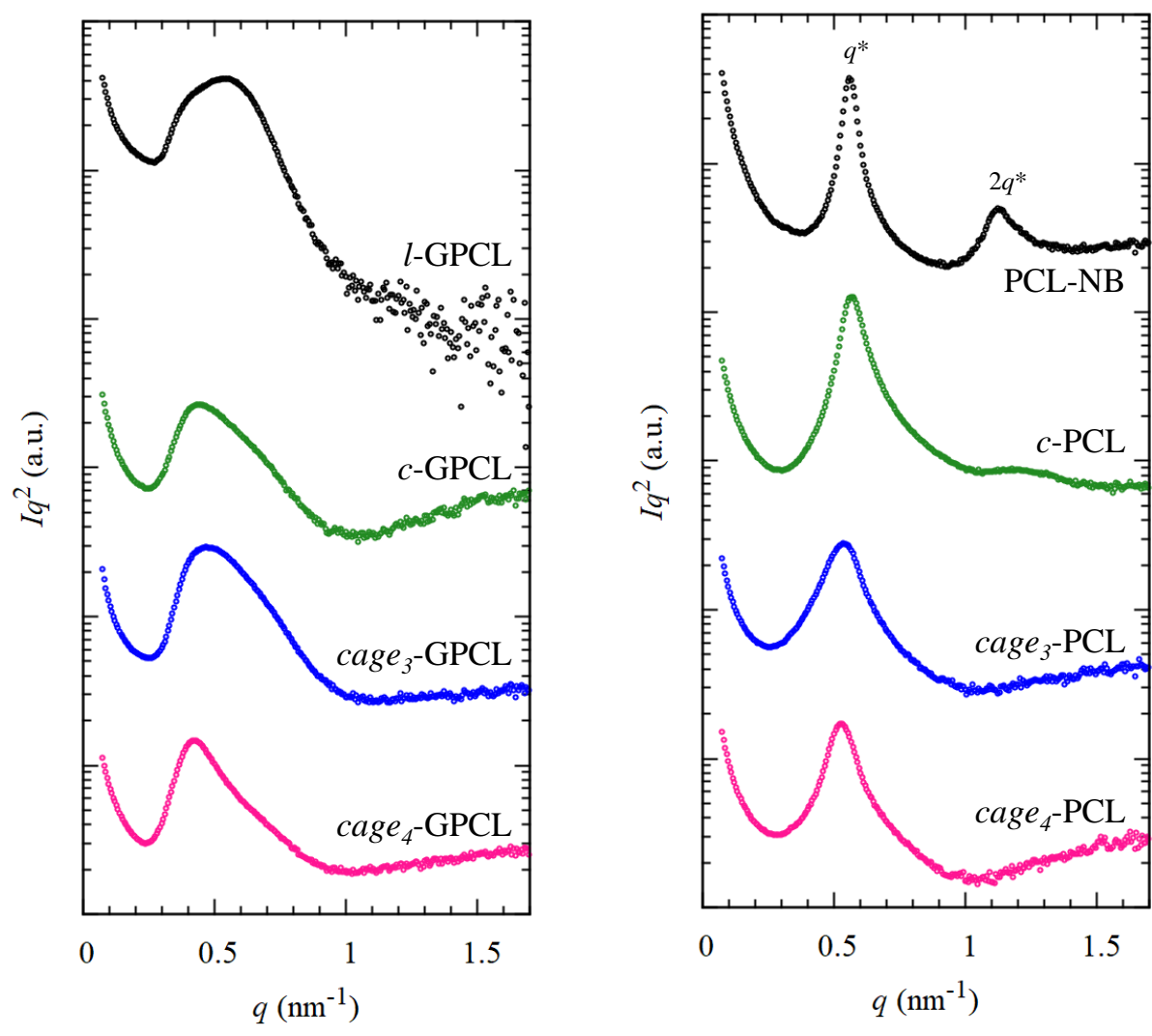

Figure S23. SAXS profiles of topological GPCLs (left) and corresponding monomeric counterparts (right). 

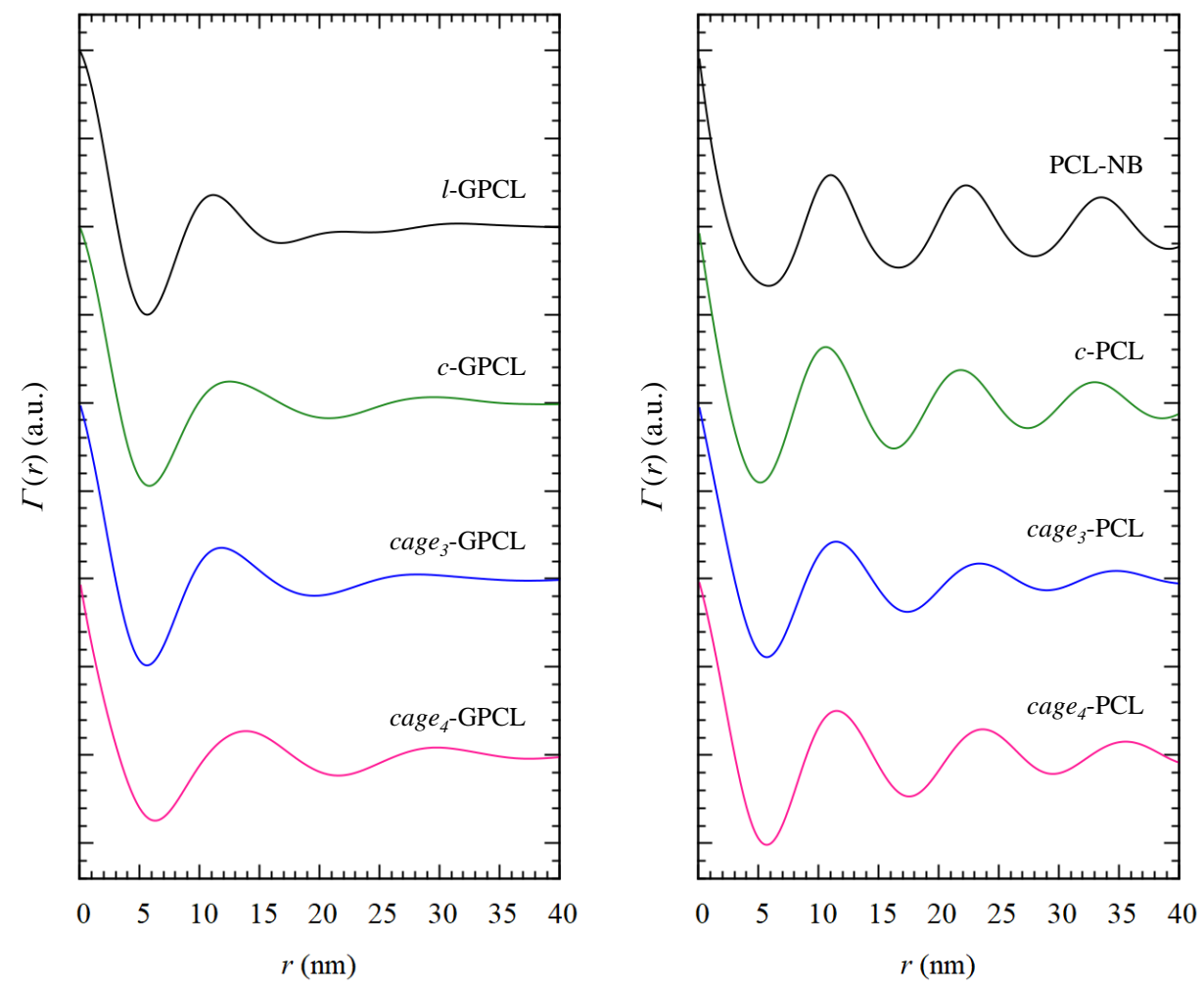

Figure S24. Correlation functions of SAXS profiles for topological GPCLs (left) and corresponding monomeric counterparts (right).

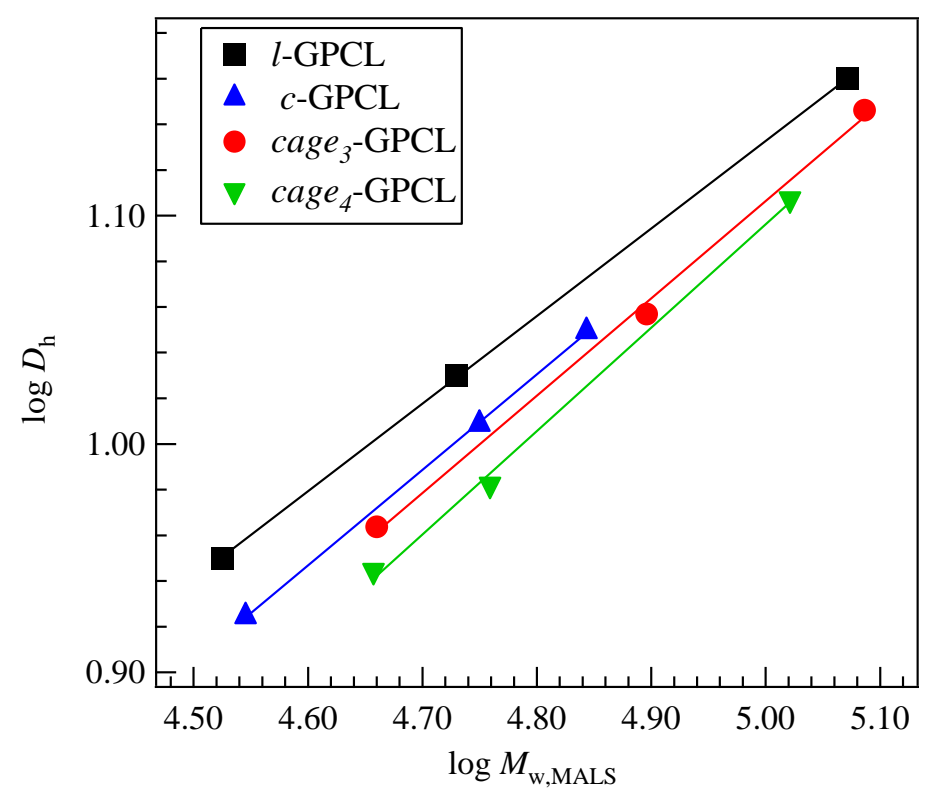

Figure S25. Double logarithmic plots of $M_{\mathrm{w}, \mathrm{MALS}}$ versus $D_{\mathrm{h}}$ for $l$-GPCLs (black), $c$-GPCLs (blue), cage $_{3}$-GPCLs (red), and cage $_{4}$-GPCLs (green). 


\section{S3. References}

(1) Mato, Y.; Honda, K.; Tajima, K.; Yamamoto, T.; Isono, T.; Satoh, T. A Versatile Synthetic Strategy for Macromolecular Cages: Intramolecular Consecutive Cyclization of Star-Shaped Polymers. Chem. Sci. 2019, 10, 440-446.

(2) Isono, T.; Sasamori, T.; Honda, K.; Mato, Y.; Yamamoto, T.; Tajima, K.; Satoh, T. Multicyclic Polymer Synthesis through Controlled/Living Cyclopolymerization of $\alpha, \omega$-DinorbornenylFunctionalized Macromonomers. Macromolecules 2018, 51, 3855-3864.

(3) Choi, T. L.; Grubbs, R. H. Controlled Living Ring-Opening-Metathesis Polymerization by a Fast-Initiating Ruthenium Catalyst. Angew. Chem. Int. Ed. 2003, 42, 1743-1746.

(4) Hoffman, J. D.; Weeks, J. J. Melting Process and the Equilibrium Melting Temperature of Polychlorotrifluoroethylene. J. Res. Natl. Bur. Stand. Sect. A 1962, 66A (1), 13-28.

(5) Vonk, C. G.; Kortleve, G. X-ray Small-Angle Scattering of Bulk Polyethylene. II. Analyses of the Scattering Curve. Colloid Polym. Sci. 1967, 220, 19-24.

(6) (a) Roe, R.-J. Methods of X-ray and Neutron Scattering in Polymer Science; Oxford University Press: New York, 2000. (b) Stribeck, N. X-Ray Scattering of Soft Matter; Springer-Verlag: Berlin, 2007.

(7) Strobl, G. R.; Schneider, M. Direct Evaluation of the Electron Density Correlation Function of Partially Crystalline Polymers. J. Polym. Sci., Polym. Phys. Ed. 1980, 18, 1343-1359. 